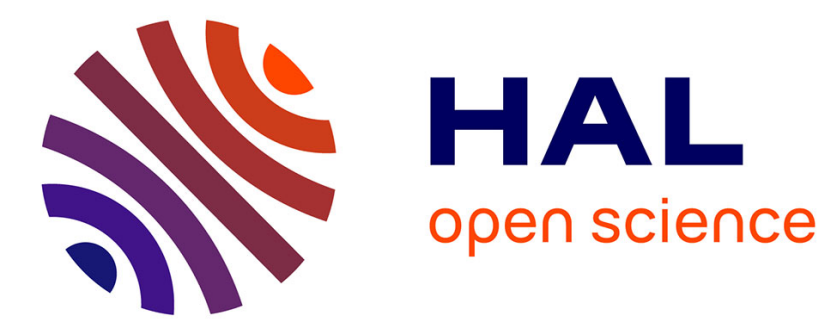

\title{
Wind measurements in Mars' middle atmosphere: IRAM Plateau de Bure interferometric CO observations
}

\author{
R. Moreno, E. Lellouch, F. Forget, T. Encrenaz, S. Guilloteau, E. Millour
}

\section{To cite this version:}

R. Moreno, E. Lellouch, F. Forget, T. Encrenaz, S. Guilloteau, et al.. Wind measurements in Mars' middle atmosphere: IRAM Plateau de Bure interferometric CO observations. Icarus, 2009, 201 (2), pp.549. 10.1016/j.icarus.2009.01.027 . hal-00533505

\section{HAL Id: hal-00533505 https://hal.science/hal-00533505}

Submitted on 7 Nov 2010

HAL is a multi-disciplinary open access archive for the deposit and dissemination of scientific research documents, whether they are published or not. The documents may come from teaching and research institutions in France or abroad, or from public or private research centers.
L'archive ouverte pluridisciplinaire HAL, est destinée au dépôt et à la diffusion de documents scientifiques de niveau recherche, publiés ou non, émanant des établissements d'enseignement et de recherche français ou étrangers, des laboratoires publics ou privés. 


\section{Accepted Manuscript}

Wind measurements in Mars' middle atmosphere: IRAM Plateau de Bure interferometric $\mathrm{CO}$ observations

R. Moreno, E. Lellouch, F. Forget, T. Encrenaz, S. Guilloteau, E. Millour

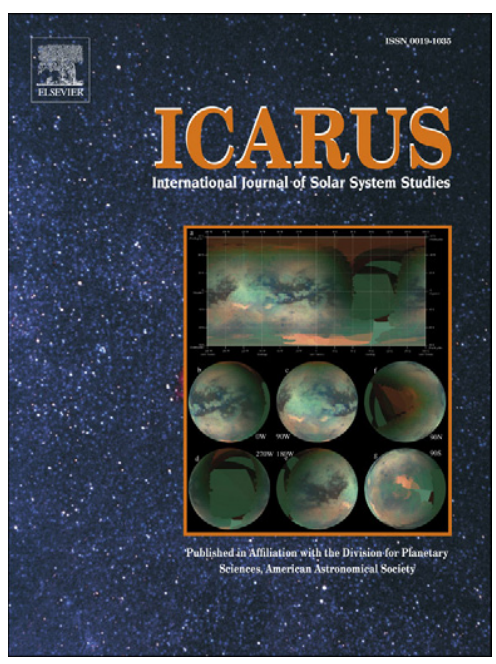

PII: $\quad$ S0019-1035(09)00061-X

DOI: $\quad$ 10.1016/j.icarus.2009.01.027

Reference: $\quad$ YICAR 8914

To appear in: Icarus

Received date: 12 June 2008

Revised date: 19 November 2008

Accepted date: 19 January 2009

Please cite this article as: R. Moreno, E. Lellouch, F. Forget, T. Encrenaz, S. Guilloteau, E. Millour, Wind measurements in Mars' middle atmosphere: IRAM Plateau de Bure interferometric CO observations, Icarus (2009), doi: 10.1016/j.icarus.2009.01.027

This is a PDF file of an unedited manuscript that has been accepted for publication. As a service to our customers we are providing this early version of the manuscript. The manuscript will undergo copyediting, typesetting, and review of the resulting proof before it is published in its final form. Please note that during the production process errors may be discovered which could affect the content, and all legal disclaimers that apply to the journal pertain. 


\title{
Wind measurements in Mars' middle atmosphere: IRAM Plateau de Bure interferometric CO observations
}

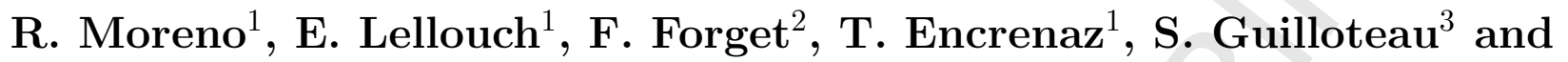 \\ E. Millour ${ }^{2}$ \\ ${ }^{1}$ LESIA, Observatoire de Paris, 92195 Meudon Cedex, France \\ E-Mail: raphael.moreno@obspm.fr \\ ${ }^{2}$ LMD, IPSL, Université Jussieu PARIS VI, Case postale 99, 4, place Jussieu, F 75252 Paris Cedex \\ 05, France \\ ${ }^{3}$ LAB - 2, Rue de l'Observatoire - BP 89 - 33270 Floirac - France
}

Submitted to Icarus on June 12th 2008

Revised on November 21th 2008

Accepted on January 27th 2009

Manuscript pages : 26

Tables : 3

Figures: 14 
Proposed Running head: Wind measurements on Mars

Editorial correspondence to:

Raphaël Moreno

LESIA, Observatoire de Paris

5, Place Jules Janssen

92195 MEUDON Cedex

FRANCE

Phone: 33-1-45077728

Fax: 33-1-45347683

E-mail: raphael.moreno@obspm.fr 


\begin{abstract}
The IRAM Plateau de Bure Interferometer has been used to map the $\mathrm{CO}(1-0)$ rotational line in Mars' middle atmosphere. Absolute winds and thermal profiles were retrieved during the 1999, 2001, 2003 and 2005 planet's oppositions. The observations sampled various seasons $\left(\mathrm{L}_{s}=143,196,262,317\right.$ and 322), and different dust situations (clear, global storm, regional storm). The absolute winds were derived by measuring directly the Doppler lineshifts. The main zonal circulation near $50 \mathrm{~km}$ is dominated by strong retrograde winds, with typical velocities of $70-170 \mathrm{~m} / \mathrm{s}$, strongly varying seasonally, latitudinally, and longitudinally (in particular between morning and evening). Comparison of the retrieved temperature with a general circulation model indicates that the model often underestimates the temperatures in the middle (20-50 $\mathrm{km}$ ) atmosphere, and overestimates them above $50 \mathrm{~km}$.
\end{abstract}

Key Words: MARS/ ATMOSPHERE, DYNAMICS 


\section{Introduction}

Characterizing the circulation of the Martian atmosphere is an essential objective to understand its present meteorology and climate, a first step to the understanding of the past climates of Mars. The lower atmosphere $(<40 \mathrm{~km})$ and middle atmosphere $(40-80 \mathrm{~km})$ of Mars appear dynamically coupled to a much higher degree than in the case of the Earth. The vertical extension of the weather phenomena is considerable, with for example Hadley cells reaching the top of the neutral atmosphere $(120 \mathrm{~km})$. The circulation in the middle atmosphere modifies the meteorology of the lower atmosphere, affecting transport and climatic processes. While the lower atmosphere circulation is well characterized, notably through extensive thermal field measurements at 0-40 km from spacecrafts (e.g. Hinson et al. 2004, Grassi et al. 2007), and succesfully reproduced by general circulation models, the upper atmosphere appears much less constrained, due to the difficulty to find suitable dynamical tracers. Thus, although models of the entire atmosphere from the surface up to the thermosphere exist (e.g. Angelats i Coll 2005, Hartogh et al. 2005), they still lack observational constraints. Heterodyne observations at millimeter or thermal infrared wavelengths are able to probe the martian middle atmosphere, providing temperature and wind measurements from spectrally resolved and Doppler-shifted molecular lines. Single-dish millimeter observations of CO constrain the temperature profile over 0-70 km (Clancy et al. 1990), and when mapped over the martian disk, have provided the first wind measurements near $50 \mathrm{~km}$ altitude (see Lellouch et al. 1991, Gillet and Lellouch 1994, Cavalié et al. 2008 from IRAM-30 m, and Clancy et al. 2006 from JCMT). Such measurements, however, are limited by their poor, diffraction-limited, spatial resolution (typically $10^{\prime \prime}$ ), enabling only an essentially hemispheric resolution of the martian disk. A much higher spatial resolution $\left(\sim 2^{\prime \prime}\right)$ is reachable in the thermal infrared (Sonnabend et al. 2006), but the difficulty is to estimate the altitude probed by the non-LTE $\mathrm{CO}_{2}$ emission used as a wind tracer. Millimeter interferometry allows one to better spatially resolve the Martian disk, and provides complete wind maps. We present here repeated measurements of the $\mathrm{CO}(1-0)$ line, obtained with the IRAM Plateau de Bure Interferometer (PdBI). From these data, temperatures and winds in the middle atmosphere around the 1999, 2001, 2003 and 2005 oppositions, were retrieved and compared with expectations from a Mars General Circulation model (Forget et al. 1999). 


\section{PdBI observations}

We observed Mars around the 1999, 2001, 2003 and 2005 oppositions with the IRAM Plateau de Bure Interferometer (PdBI). Between May 1999- Sep. 2003, the PdBI consisted of 5 antennas of 15-m, and was extended to a sixth antenna after Dec. 2003. Observations were targetted at the $\mathrm{CO}(1-0)$ rotational line at $115.271 \mathrm{GHz}$. Although the $\mathrm{CO}(2-1)$ line at $230.538 \mathrm{GHz}$, previously used for IRAM-30m wind measurements, is stronger than $\mathrm{CO}(1-0)$ and provides a twice better wind sensitivity for a given $\mathrm{S} / \mathrm{N}$, the choice of the $\mathrm{CO}(1-0)$ line was dictated by the need to avoid the spatial filtering of the interferometer due to the lack of short spacing visibilities at $230 \mathrm{GHz}$. Indeed even with the most compact configuration, the typical 15" Mars' angular diameter was too much resolved for the $\mathrm{CO}(2-1)$ line. Depending on the year, the antennas of the interferometer were in compact configuration $\mathrm{D}$ or $\mathrm{C}$, with baselines ranging from 15-75 m (D) and 20-150 m (C), providing a synthesized beam with equivalent diameter in the range 3.5-7" in $\mathrm{CO}(1-0)$. Depending on the period, the angular diameter of Mars was between $9.5^{\prime \prime}$ and $23^{\prime \prime}$, and the synthesized beam typically resolved Mars in $4 \times 4$ independent points (Table 1). For the first year (1999), observations were actually acquired over 4 different days, and the data combined in the reduction phase as detailed below. In 2003, observations were obtained at two different periods, about 3 weeks and 3 months, respectively, after the 2003 Aug. 27 opposition. Altogether, the observations sampled various seasons $\left(\mathrm{L}_{s}=143,196\right.$, 262, 317 and 322), and as discussed later different dust situations (clear, global storm, regional storm). We used a variety of backends, affording a spectral resolution up to $40 \mathrm{kHz}$, suitable to resolve the line core $(\sim 400 \mathrm{kHz}$ wide), and therefore to measure and map Doppler shifts

of about $40 \mathrm{kHz}$ at the planet limb. On a typical day, Mars observations lasted $\sim 6$ hours to perform the aperture synthesis. They were interrupted every 20 minutes, so as to perform an amplitude and phase calibration sequence on a nearby quasar. Pointing and focus checks were also regularly performed. Throughout the observations, weather conditions (water vapor humidity) were monitored with a water vapor radiometer at $22 \mathrm{GHz}$, except in 1999 where the total power at $230 \mathrm{GHz}$ was used. These radiometer measurements were used to improve the atmospheric phase correction. 


\section{Data reduction}

The data reduction and image processing were performed using the GILDAS ${ }^{1}$ packages developed at IRAM. A standard calibration was performed with the GILDAS/CLIC interferometric data reduction package. The essential elements of the reduction are : (i) calibration of the instrument bandpass (ii) correction of amplitude and phase variations with time using quasar measurements, and (iii) calibration of the flux scale. The measured phase rms was typically $20^{\circ}$ and the relative amplitude calibration with time about $5 \%$. The measured visibilities (i.e. the Fourier transform of the image) were then extracted in a uv-table. A rotation of the polar axis direction was directly applied in the uv-table, in order to present maps with the North pole on the top of the image.

The second step was to self-calibrate the visibilities in phase and amplitude against a Mars continuum model described in the next section. This baseline-based self-calibration improves image quality and provides a relative flux accuracy below 1-2\%, while the absolute flux accuracy is defined by the absolute accuracy of the continuum model (i.e. $5 \%$ in our case).

Image processing was done using the GILDAS/MAPPING aperture synthesis imaging package. The continuum was subtracted before creating the images, to improve image dynamics. The dirty images were deconvolved using a CLARK algorithm, providing a CLEAN line-contrast image for all spectral channels. These CLEAN spectral maps were used for the Doppler shift measurements. To obtain the line-to-continuum maps needed to perform the thermal structure analysis, the CLEAN line-contrast map was divided by the CLEAN continuum map. Due to the self-calibration, the CLEAN continuum map is nearly identical to the CLEAN model map.

\subsection{Continuum modelling : determination of surface roughness}

For each date of observation, a Mars continuum map was computed, based on a radiative transfer model of the martian subsurface. Essentially, this model ${ }^{2}$ uses General Climate Model predictions of martian surface and sub-surface temperatures ${ }^{3}$, solves the radiative transfer

\footnotetext{
${ }^{1}$ web adress : www.iram.fr/IRAMFR/GILDAS/

2 available at www.lesia.obspm.fr/ lellouch/mars

${ }^{3}$ see www-mars.lmd.jussieu.fr/
} 
equation within the subsurface (e.g. Rudy et al. 1987, Muhleman and Berge 1991), on a $50 \times 50$ points horizontal grid. Once the epoch and geometrical parameters (subearth latitude, longitude and local time) are fixed, the parameters of the models are (i) the absorption length $\mathrm{k}_{\lambda}$, expressed in units of the wavelength $\lambda$ (ii) the surface dielectric constant $\epsilon$ and (iii) the surface roughness (degrees), expressed as the rms dispersion of the local surface slope angle. The above parameters, particularly the dielectric constant and the surface roughness, have correlated effects on the distribution of brightness temperatures, so all three parameters cannot be retrieved univoquely. Following Muhleman et al. (1991), we fixed $\mathrm{k}_{\lambda}=12 \lambda$, and $\epsilon=2.25$, and selected the surface roughness as the free, adjustable parameter. Continuum brightness temperature maps calculated in this manner were used to generate synthetic visibilities (for the same uv sampling as in the actual data) and compared to the measurements.

On the example of Dec. 16, 2003, Fig. 1 shows a comparison of the modelled continuum visibilities for various values of the surface roughness, and the measured visibilities (of course, before self-calibration). The complex structure of the amplitude of the visibilities versus baseline length (UV radius) is generally accounted for by the continuum model. Note how in particular the multiple branches in the vicinity of zero-crossing radii, associated to the complex latitude - and local-time - dependent distribution of surface temperatures. For all observation periods, fits of the continuum visibilities - in particular in the vicinity of the so called zero-crossing, where the amplitude is close to 0 - resulted in a best-fit value of the surface roughness. Results for the individual periods are given in Table 2. The mean derived surface roughness is $15^{\circ} \pm 10^{\circ}$.

\section{Wind measurements}

Beam-convolved, line-of-sight projected, wind velocities were obtained from centroiding of the $\mathrm{CO}(1-0)$ Doppler line core. For this, starting from the line-contrast map, a baseline polynomial fit of the linewings (over $\pm[1-10] \mathrm{km} / \mathrm{s}$ from line center) was performed and subtracted from the data. A gaussian fit of the resulting line core was then performed over the central \pm 1 $\mathrm{km} / \mathrm{s}$. The fitting interval was choosen to optimize the probing at the highest altitudes derived by the wind weighting function (see below). The precision on the Doppler shift determined in this manner is typically $15-25 \mathrm{~m} / \mathrm{s}$. An example of line fitting is shown in Fig. 2. We have 
tested other baseline fit intervals (over $\pm[5-30] \mathrm{km} / \mathrm{s}$ from line center) and other gaussian fit interval (over $\pm 3 \mathrm{~km} / \mathrm{s}$ ). The Doppler shifts for these less optimized cases have almost the same central value, and the rms increases by only $5 \mathrm{~m} / \mathrm{s}$, which is much less than the average precision, thus negligeable. A data selection was performed to keep only the spectrum with a flux signal-to-noise ratio greater 4 .

The measured Doppler shifts represent the sum of the planet solid rotation and the horizontal winds, projected on the line-of-sight and convolved with the synthesized beam. The contribution of the planet solid rotation was computed (from our square two-dimensional finite element model, Moreno et al. 2001) and subtracted in order to derive the Doppler wind.

The resulting wind maps are shown in Fig. 4, 8, 10, 11, 12. Once again, these are beamconvolved and line-of-sight projected winds. To provide an idea of the associated horizontal winds, we have also "deprojected" these winds for a few characteristics points (north and south, western and eastern limb) as detailed below. Results are indicated in Table 3.

The sounded altitude is estimated by calculating wind weighting functions, as defined by Théodore et al. (1993). Essentially, contribution functions are calculated at all frequencies over the wind fitting spectral window $( \pm 1 \mathrm{~km} / \mathrm{s})$, weighted by the local slope (derivative) of the spectrum and averaged. Mathematically, the wind weighting function $(W W F)$ can be defined as :

$$
W W F(z)=\int_{\nu_{0}-\Delta \nu}^{\nu_{0}+\Delta \nu} C F(\nu, z) \times\left|\frac{d I(\nu)}{d \nu}\right| \times d \nu
$$

where $I(\nu)$ is the radiance and $C F(\nu, z)$ is the contribution function :

$$
C F(\nu, z)=B_{\nu}(T(z)) \times \frac{d e^{-\tau(\nu)}}{d z}
$$

and $d \nu$ runs over the fitting interval $(\Delta \nu=400 \mathrm{kHz}$ in our case, i.e. $1 \mathrm{~km} / \mathrm{s}), B_{\nu}$ is the Planck function at temperature $T, \tau$ is the opacity in the line-of-sight, $z$ is the altitude. Since most of the wind information comes from the limbs, these contribution functions were calculated for the geometry of a limb point, using a typical temperature profile and a Mars to beam diameter ratio of 4. As shown in Fig. 3, the altitude probed for a nadir geometry would be $\sim 4 \mathrm{~km}$ only lower. The altitude probed at the limb is $\sim 53 \pm 13 \mathrm{~km}$ (where the range corresponds to altitudes at half-width of the WWF). This altitude is only approximate, however, because it depends on the way monochromatic contribution functions are averaged (see e.g. Théodore et 
al. 1993) and varies with the target point, in relation with varying temperature profile and airmass conditions.

\section{Results and comparisons with global climate pre- dictions}

\subsection{Direct model}

To interpret our wind measurements and compare them with predictions based on global climate models, we developed a so-called direct model by coupling outputs from the Martian Climate Database (MCD, v4.1) to a standard radiative transfer code. The MCD is a database describing the climate and environment of the Martian atmosphere, derived from the LMD martian general circulation model (Forget et al. 1999, Lewis et al. 1999). Essentially, for each of the observed appearances of Mars, we partitioned the martian disk in a fine, regular grid (100 points across a martian diameter). At each point of the grid, the relevant parameters (latitude, longitude, local time, season) were calculated. The MCD database was then interrogated, providing the vertical profiles of pressure, temperature and wind coordinates (zonal and meridional). For points within the solid planet, the airmass was calculated by using the Chapman function, to account for the spherical geometry. Radiative transfer at the limb was calculated with a full geometrical treatment (i.e. calculating the path in each layer in an exact manner) and with a step of $1 \mathrm{~km}$ in the tangent altitude. Points close to but outside the solid disk were also considered, in order to include limb viewing in the radiative transfer. For those points, we used temperature and wind conditions of the closest disk point. In all the calculations, we used the Martian Year 24 (as defined in Clancy et al. 2000) dust and average solar flux scenario, except in the case of the July 2001 observation, for which a "dust storm" (optical depth =4) case was used. The Martian Year 24 dust scenario is based on a climatology of the dust as observed by Mars Global Surveyor TES between 1999 and June 2001 (Smith, 2004), a period thought to be typical. Because our observations usually lasted for several hours, during which period Mars rotated significantly, we extracted the atmospheric parameters several times (once per hour) according to the variation of the central meridian longitude, and averaged them before use in 
the radiative transfer code. A synthetic $\mathrm{CO}(1-0)$ line was then calculated at each point of the fine grid, accounting for the appropriate airmass of each layer in vertical or horizontal viewing. To include the effect of winds, the line-of-sight velocity was calculated from the projection of the zonal and meridional wind components to the observer direction. This Doppler velocity was then included level-by-level in the calculation of the absorption coefficient. A CO mixing ratio of $10^{-3}$ was used in the lower atmosphere. This is slightly larger than the "canonical" $8 \times 10^{-4}$ value, but more in line with findings from recent infrared observations (Krasnopolsky 2003, 2007). Above $\sim 40 \mathrm{~km}, \mathrm{CO}$ increases with altitude due to its high-altitude production and lifetime shorter than atmospheric mixing time, and we used the CO profile from Théodore et al. (1993). No horizontal (diurnal, latitudinal) variations of CO were considered. Although such variations are indicated by the above infrared observations and may be expected from general circulation models, the precise horizontal (and even vertical) CO profile is to first order inconsequential to interpret our observations in terms of winds and atmospheric structure in the middle atmosphere. To calculate the continuum emission, we used the continuum model determined from the analysis of the continuum visibilities, as described previously. The local CO lines were finally convolved by the appropriate beam geometry (including elliptical shape, orientation and gaussian response over any distance from beam center) on an array adequately matched to the spatial resolution of the observations. This provided us with "synthetic" data that could be compared directly to the observations. At each point of the array, the synthetic spectrum was fit over its central $\pm 1 \mathrm{~km} / \mathrm{s}$, by a "template" spectrum calculated in the same manner but in which the winds were forced to zero. This allowed us to determine the wind field that would result from such "synthetic data", i.e. the wind field we would observe if the MCD database gave a perfect representation of the wind/temperature field in the martian atmosphere. This method provides the most rigorous comparison between observations and models, and in particular, is superior to a comparison of the measured wind field with the modelled wind field averaged over some altitude range. As discussed above, the level probed for the winds is typically indicated by the wind weighting functions, and corresponds to $\sim 35-65 \mathrm{~km}$ at the limb, for the central $\pm 1 \mathrm{~km} / \mathrm{s}$ of the $\mathrm{CO}(1-0)$ line, but this varies across the disk (e.g. with a nadir viewing we probe at lower altitude). Besides the calculation of the wind field expected from GCM predictions, the direct model allows us to calculate synthetic lineshapes and to compare 
them with the observed profiles.

\subsection{Thermal profile retrieval}

As will be shown, in most cases, the synthetic spectra calculated with the direct model do not match perfectly the observed lineshapes, indicative or more or less important departures between predicted and actual thermal profiles. To get a handle on these differences, we performed temperature inversions. In this procedure, a single temperature profile can be retrieved for each beam position. Given the relatively coarse spatial resolution of the data (which makes the direct model a more rigorous approach), this clearly limits the physical meaning of the retrieved thermal profiles, but the comparison with the beam-convolved temperature profiles returned from the MCD is still instructive. Weighting functions (see e.g. Clancy et al. 1990, Lellouch et al. 1991b) indicates that the $\mathrm{CO}(1-0)$ line absorption core within $\sim 20 \mathrm{MHz}$ from line center probes the middle atmosphere from $\sim 15 \mathrm{~km}$ up to $\sim 70 \mathrm{~km}$. More distant linewings probe the lower atmosphere, but there, the accuracy of the temperature retrievals is limited by the quality of the instrumental baselines and the precise knowledge of the $\mathrm{CO}$ mixing ratio. We therefore focussed on the absorption cores, by expressing the measured fluxes in terms of a relative linedepth with respect to a "pseudo-continuum" at $\pm 17 \mathrm{MHz}$ from line center. For each beam position, the beam-convolved surface temperature was calculated from MCD and kept fixed, and the line profile was inverted in terms of the temperature profile from an iterative Chahine-type algorithm, used previously by Lellouch et al. (1991b) and Théodore et al. (1993). In this method, a small number of frequency points are selected and assigned to representative altitude levels located near the maximum of the associated weighting functions. The temperature at these levels was found iteratively, with some smoothing of the temperature profile at each iteration, and by forcing the retrieved thermal profile to join the near-surface temperature of the a priori (i.e. GCM-derived) profile.

\subsection{Wind and temperature results}

For each of the 5 observing periods, results are presented in the form of four figures showing respectively: 
- the measured line-of-sight winds

- the line-of-sight winds expected from GCM predictions

- the observed CO lines in the central $\pm 10 \mathrm{MHz}$ (black lines) along with (i) the lines expected from the direct model (red lines) and (ii) the fit to the observed lines with the temperature inversion (green lines)

- the comparison of the beam-averaged thermal profile based on GCM (red) with the beamintegrated retrieved profile (green)

Note that rather than presenting the observations in chronological order, we organize the discussion according to dust content and Martian season. We take information on the dust content from MGS/TES and Mars Odyssey/THEMIS measurements (Smith et al. 2004, Lillis et al. 2008).

\subsubsection{Normal dust $(\tau=0.3)$ - September $2003\left(\mathrm{~L}_{s}=262\right)$}

Fig. 4, 5, 6, 7 shows results near Southern summer solstice $\left(\mathrm{L}_{s}=262\right)$ observed in September 2003. For the first period, and to enhance the visibilities, the four figures are shown separately and in large format. For such a period, the GCM predicts a mostly retrograde flow with core near $20^{\circ} \mathrm{S}$ latitude and core speed of about $120 \mathrm{~m} / \mathrm{s}$. Although a strong prograde jet is also present in the winter hemisphere, it becomes important only at latitudes higher than $\sim 50 \mathrm{~N}$. Given our observing geometry with a subearth point at $20^{\circ} \mathrm{S}$, this prograde wind should be essentially invisible in our observations. Indeed the GCM-predicted wind map shows strong retrograde winds, with $-60 \mathrm{~m} / \mathrm{s}$ (approaching) winds at the equatorial western (evening) limb and $+90 \mathrm{~m} / \mathrm{s}$ (recessing) at the eastern (morning) limb. (We adopt the astronomical convention for East and West). This feature closely matches the observed winds at low latitudes. Note however that the observed winds also show a strong prograde wind at mid-Northern $\left(\sim 45^{\circ} \mathrm{N}\right)$ latitudes. This suggests that the winter prograde jet was present at somewhat lower latitudes than expected by the GCM. In the southern polar regions, the circulation is characterized by recessing winds on both the morning and evening side. This could be explained by a global transpolar flow from the dayside of the planet to the nightside. CO lineshapes calculated for the direct model agree generally well with the observations. The agreement is particularly good at low latitudes, 
where GCM-derived and retrieved thermal profiles coincide quite well in shape up to at least $60 \mathrm{~km}$. At high Southern latitudes, and on the morning side of the high Northern latitudes, the synthetic lines appear slightly too narrow, suggesting that the temperature gradient above $20 \mathrm{~km}$ altitude is a somewhat underestimated by the model. Nonetheless, the GCM-predicted winds and temperatures agree overall reasonably well with the observations. From similar $\mathrm{CO}(3-2)$ single-dish measurements, Clancy et al. (2006) also find good agreement between their retrieved thermal profile and the MGS-TES temperature profile for the same epoch, local time and location.

\subsubsection{Low dust $(\tau=0.1)$ - May $1999\left(\mathrm{~L}_{s}=143\right)$}

Fig. 8 shows results for late Northern summer $\left(\mathrm{L}_{s}=143\right)$ observed in May 1999. Fig. 9 shows the Doppler winds expected from GCM predictions in the absence of beam convolution. At $\mathrm{L}_{s}$ $=143$, the GCM predicts a moderate (maximum core flow of $\sim 70 \mathrm{~m} / \mathrm{s}$ ) retrograde flow centered on the Equator, and a stronger prograde jet at high Southern latitudes. As the latter is poorly seen in our observing geometry, the convolved and projected circulation appears to consist of weak $(20-30 \mathrm{~m} / \mathrm{s})$ retrograde winds. The observed winds are considerably stronger $(80-100 \mathrm{~m} / \mathrm{s}$ at western limb) than anticipated, and show a significant East/West asymetry at low latitudes and in the Northern hemisphere.

The GCM-temperature profiles do not allow a good match of the observed lineshapes. The largest problems occur in the winter (Southern) hemisphere and at low latitudes, where the narrow absorption cores are not reproduced. In contrast, the broader lines at high and polar northern latitudes are well accounted for by the direct model. Analysis of the temperature profile show that the GCM slightly underestimates temperatures between 0 and $50 \mathrm{~km}$ in the southern hemisphere, most likely because of dust or cloud activity not well accounted for in the GCM. These observed warmer temperatures correspond to a more intense meridional circulation and thus more intense zonal jets (Haberle et al. 1993). This may partly explain the observed difference between modeled winds and observations. It is interesting to note that the maximum temperature discrepancy between model and observations is observed in the afternoon in the tropics, around 30-40 km altitude. At this particular season and time, this warm bias may be attributed to the presence of clouds which have recently been shown to warm the atmosphere 
by more than $10 \mathrm{~K}$ (Wilson et al 2008) probably because of their absorption of the upward thermal radiation.

Above $50 \mathrm{~km}$, the GCM seems to overestimate the temperatures. Such a discrepancy is consistent with the observations obtained by the UV spectrometer SPICAM on Mars Express using stellar occultation technique (Forget et al. 2008). The model error is thought to result from an underestimation of the infrared cooling rate in the $\mathrm{CO}_{2} 15$ microns band in non thermal equilibrium conditions.

\subsubsection{Regional dust storm $(\tau=0.5)$ - December $2003\left(\mathrm{~L}_{s}=317\right)$}

Fig. 10 shows results for late Southern summer $\left(\mathrm{L}_{s}=317\right)$ observed in December 2003. For this period, the data show a very strong retrograde flow, with a large East/West asymetry $(-185 \mathrm{~m} / \mathrm{s}$ at equatorial West limb and $+10 \mathrm{~m} / \mathrm{s}$ at East limb). The retrograde flow appears to decrease with latitude on the West limb, but to increase on the East limb, at least in the Southern hemisphere, where the East/West asymetry is considerably subdued. This appears to be severaly at odds with GCM predictions. Essentially the GCM winds at this season (for a standard MY24 dust scenario) have a structure similar to that predicted for $\mathrm{L}_{s}=262$, except that the Southern hemisphere retrograde flow is weaker and the prograde wind in the Northern hemisphere extends to lower latitudes. As a consequence, when convolved with the instrumental beam, only weak retrograde winds $(\sim 30 \mathrm{~m} / \mathrm{s})$ would be expected, in striking contrast with the strong and asymetric observed winds. Fig. 10 shows that the GCM-derived temperature profiles do not match the observed lineshapes. At most points, lines derived from the direct model are too broad at $\pm 2-10 \mathrm{MHz}$ from line center and conversely lack the narrow absorption core present in the central $\pm 2 \mathrm{MHz}$ of the data. Temperature comparisons indicate that the GCM temperatures decrease too much with altitude over $\sim 30-50 \mathrm{~km}$ and not enough above $50 \mathrm{~km}$. As for the $\mathrm{L}_{s}=143$ season, the discrepancy is particularly striking near low-latitude limb points and in the winter (now Northern) hemisphere. For those points, over the $30-50 \mathrm{~km}$ range, temperature retrievals suggest a broad quasi-isothermal region in the summer hemisphere and a temperature inversion in the northern winter hemisphere. The possible occurence of a temperature inversion in the winter hemisphere was reported by Théodore et al. (1993) from similar data obtained at $\mathrm{L}_{s}=279$. Most of these differences between model and observations 
can probably be explained by the fact that $\mathrm{L}_{s}=317$ in 2003 corresponds to the beginning of a dust storm event much stronger than what happened during the reference year (Martian 24, 1999-2000) used to estimate the amount of dust in the atmosphere in the GCM (Smith et al. 2006, Wang et al. 2005). High dust content at this season is expected to warm the low and mid atmosphere, strongly enhance the Hadley circulation, and increase the warming of the middle atmosphere (Forget et al. 1999). The latitudinal and vertical structure of the differences between model and observations are in good agreement with this explanation.

\subsubsection{Regional dust storm $(\tau=0.2)$ - November $2005\left(\mathrm{~L}_{s}=322\right)$}

The November 2005 observations occurred essentially at the same season (late Southern summer, $\mathrm{L}_{s}=322$, Martian year 25) as for December $2003\left(\mathrm{~L}_{s}=317\right.$, Martian Year 26). Again, and in contradiction with GCM predictions for this season, the data show a very strong retrograde flow with a large East/West assymetry (Fig. 11). Unlike in December 2003, however, the wind is stronger on the Equatorial East limb $(210 \mathrm{~m} / \mathrm{s})$ than on the West limb $(-120 \mathrm{~m} / \mathrm{s})$. As the local time of the subearth point significantly differs for the two observations (11:45 am in Nov. 2005 vs 9:00 am in Dec. 2003), the different wind field might reflect a local time forcing. In this case, the equatorial velocity would abruptly rise from $10 \mathrm{~m} / \mathrm{s}$ at 6 am to $210 \mathrm{~m} / \mathrm{s}$ at $9 \mathrm{pm}$, and then slowly decrease from $180 \mathrm{~m} / \mathrm{s}$ at $3 \mathrm{pm}$ and $120 \mathrm{~m} / \mathrm{s}$ at $6 \mathrm{pm}$.

However, as mentionned above, another aspect to consider is that both epochs correspond to the $\mathrm{L}_{s}=315-330$ discrete storm season (e.g. Wang et al. 2005, 2007) during which the global dust loading is increased (Smith 2004), but not in a necessarily repeatable manner. Themis (Mars Odyssey) data indicate that the November 2005 atmosphere (Martian year 26) was probably dustier than during Martian year 24 at the same season (M.D. Smith, personal communication). Synthetic spectra generated with the GCM temperature field for the standard (MY 24 dust) scenario produce CO lines narrower and shallower than observed. This reflects a warmer than predicted atmosphere throughout the planet, as expected. This result agrees with

findings by Clancy et al. (2006) and Cavalié et al. (2008) from nearly simultanenous (Nov. 4, 2005 and Oct. 31, 2005, respectively) single-dish CO (3-2) and (2-1) measurements. As before, the temperature difference may partly explain the observed difference in circulation between model and observations. 


\subsubsection{Global dust storm $(\tau=4)$ - July $2001\left(\mathrm{~L}_{s}=197\right)$}

The July 2001 period $\left(\mathrm{L}_{s}=197\right)$ was characterized by a global dust storm, well documented by MGS observations (Strausberg et al. 2005, Smith 2002). For July 17, 2001 MGS/TES observations (Smith 2002) indicate a $9 \mu \mathrm{m}$ optical depth in excess of 2 in most of the Southern hemisphere. For this season and for dust storm condition (dust optical depth $=4$ ), the GCM predicts two prograde jets (core speed $80-100 \mathrm{~m} / \mathrm{s}$ ) centered at $\sim 60^{\circ} \mathrm{N}$ and $70^{\circ} \mathrm{S}$ superimposed on a low latitude retrograde flow. This structure remains visible even after convolution with our (elongated in the North-South direction) beam, but the anticipated projected winds do not exceed $40 \mathrm{~m} / \mathrm{s}$ (prograde, near both poles) and $30 \mathrm{~m} / \mathrm{s}$ (retrograde, near equatorial west limb) (Fig. 12). While the observed wind field follows, to some extent, this structure, the most striking difference is the fact that the observed Northern hemisphere jet is $\sim 3-4$ times stronger than expected. Interestingly, meridional winds near the South Pole are observed as predicted. The observed lineshapes are very different from those observed in any other period, showing virtually no sign of absorption except for the central $\pm 3 \mathrm{MHz}$. This results from the fact that the dust storm atmosphere is globally much more isothermal, as shown by the red lines in Fig. 12. Interestingly, while the GCM thermal profiles allow us to nicely reproduce the lines southward of $\sim 20^{\circ} \mathrm{S}$, the discrepancy become progressively worse when going into the Northern hemisphere, where the actual data show that the temperature profiles are not isothermal to as high altitudes as predicted. This is also where the observed and predicted wind field appear to be the most different.

\section{Comparison with other wind measurements}

\subsection{IRAM-30 m measurements}

Lellouch et al. (1991a) obtained the first wind measurements in Mars' middle atmosphere, using the $\mathrm{CO}(2-1)$ line observed with the IRAM-30 m telescope in September 1988, and revealing a strong retrograde flow at Southern summer solstice $\left(\mathrm{L}_{s}=279\right)$. Since then, the same facility has been used in repeated occasions to monitor the wind field over 40-70 km. Observations were acquired during the 1990, 1992/1993, 1997, 1999, 2001, 2003 and 2005 Mars' oppositions, 
and span a variety of solar longitudes (Table 3). Data from 1990 to 1999 have been presented in partial form by Gillet and Lellouch (1994) and Jegou (2003), but so far were not published extensively. Fig. 14 presents a synthetic view of all these wind measurements, except for the 2001 and 2005 data, which are described by Cavalié et al. (2008). Single-dish observations make use of a "point-by-point" mapping technique, in which spectra are accumulated on a sequence of individual beam positions. Fig. 13 shows the line-of-sight wind velocities, measured by gaussian fitting of the line core over $\pm 1 \mathrm{MHz}$ from the $\mathrm{CO}(2-1)$ rest frequency $(230538.000 \mathrm{MHz})$, and corrected for Mars' surface rotation. Inspection of the continuum levels allows one to estimate the magnitude and direction of the pointing errors. These pointing errors, which generally lie within 2 arcsec, are taken into account in Fig. 13. Fig. 13 clearly indicates that for all observing periods, the flow is primarily retrograde. Fitting the observed wind velocities with a simple solidbody rotation model, in which the retrograde equatorial velocity $\mathrm{v}_{e q}$ is the only free parameter, indicates best fit values of $\mathrm{v}_{e q}$ varying from $80 \pm 25 \mathrm{~m} / \mathrm{s}$ in August 2003 to $170 \pm 70 \mathrm{~m} / \mathrm{s}$ in April 1997 (Table 3). Nonetheless, in most cases, such a simple model does not allow a good fit of the measurements, which in spite of their modest spatial resolution (typically 10 arcsec), show considerably more structure than a solid-body rotation. To get a handle of this, Table 3 and Fig. 14 provides, for each observing period, the "deprojected wind" for several characteristic points (the Equatorial limbs, and when available, the mid-Northern and mid-Southern latitude limbs). For a given beam position, the deprojected wind is the value of the zonal wind (assumed to be constant over the planet), that would, after projection to the observer and convolution by the telescope beam, yield the observed line-of-sight velocity. As is clear in Fig. 14, the deprojected wind appears, at the resolution of the measurements, retrograde in most places. In particular, equatorial winds appear retrograde in all situations. For mid-latitudes, the only cases where prograde winds are present in the 30-m data are near summer Southern Solstice, as observed in Sept. $1988\left(\mathrm{~L}_{s}=279\right)$ and Aug. $2005\left(\mathrm{~L}_{s}=241\right)$. The other striking result of Fig. 14 is the often strong limb-to-limb (i.e. morning to evening) variation in the deprojected velocity, a behaviour reminiscent of the PdBI wind fields. Note that with the $30 \mathrm{~m}$ data, when the planet diameter was the largest during the 2003 opposition, Cavalié et al. (2008) also measured significant limb-to-limb variations up to $\sim 50 \mathrm{~m} / \mathrm{s}$. The measured equatorial limb-tolimb variation during normal dust conditions is about $10-70 \mathrm{~m} / \mathrm{s}$, but is higher $(50-230 \mathrm{~m} / \mathrm{s})$ 
during dust storm or regional dust storm conditions. The GCM predictions with the MY24 dust scenario show only limited $(10-20 \mathrm{~m} / \mathrm{s})$ morning-evening variation (between local time $6 \mathrm{~h}$ and 18h, see Fig. 14). The same behaviour is true even at higher altitude (e.g. $65 \mathrm{~km}$ ) and if we compare between local time $4 \mathrm{~h}$ and $16 \mathrm{~h}$. However, the GCM prediction in dust storm conditions indicates stronger morning-evening variations $(50-200 \mathrm{~m} / \mathrm{s})$, which is more compatible with our measurements. Note that the sign and magnitude of the East-West difference does not show any obvious trend with $\mathrm{L}_{s}$. Plotting the deprojected winds vs. the local time of the subearth point does not reveal clear trends either. This suggests the existence of large diurnal variability, though in a somewhat erratic manner.

\subsection{Other measurements}

While our dataset, augmented by the 2001 and 2005 observations presented by Cavalié et al. (2008), represents the largest body of wind measurements in Mars' middle atmosphere, other observations are available. Clancy et al. (2006) present winds deduced from ${ }^{12} \mathrm{CO}(3-2)$ and ${ }^{13} \mathrm{CO}(3-2)$ JCMT submillimeter observations on two dates in August-September 2003, probing respectively the $\sim 50-80 \mathrm{~km}$ and $30-40 \mathrm{~km}$ ranges. Again, strong retrograde winds are found. On one occasion (Aug. 28, 2003, $L_{s}=251$ ), the ${ }^{12} \mathrm{CO}(3-2)$ measurements show comparable (beam-integrated) $140-150 \mathrm{~m} / \mathrm{s}$ winds on the East and West limb. When the geometry and beam convolution are taken into account, this probably indicates deprojected winds in excess of $250 \mathrm{~m} / \mathrm{s}$. On Sept. 4, 2003, the data indicate a significant east/west asymetry, with winds stronger by $\sim 50 \mathrm{~m} / \mathrm{s}$ on the evening limb than on the morning. Finally, winds inferred from ${ }^{13} \mathrm{CO}(3-2)$ are apparently even stronger, reaching beam-integrated values of $\sim 200 \mathrm{~m} / \mathrm{s}$. While not fully consistent with our own results (as indicated in Table 3, for Sept. 1, 2003, we determine deprojected winds measured of $\sim 100-130 \mathrm{~m} / \mathrm{s}$ only, except on the evening limb at mid-Southern latitudes where they reach $190 \mathrm{~m} / \mathrm{s}$ ), the Clancy et al. (2006) results also point to the existence of very strong and longitudinally or diurnally variable winds. Additional, high spatial resolution, wind measurements were obtained from 10- $\mu \mathrm{m}$ heterodyne spectroscopy of $\mathrm{CO}_{2}$ non-LTE emission, thought to originate in the $50-90 \mathrm{~km}$ altitude range. Initial observations by Sonnabend et al. (2005) indicated a retrograde $74 \pm 22 \mathrm{~m} / \mathrm{s}$ at $20 \mathrm{~N}$ for $\mathrm{L}_{s}=308$. A more complete study, performed in December $2005\left(\mathrm{~L}_{s}=335-338\right)$ allowed Sonnabend et al. (2006) to derive a lat- 
itudinal profile of wind. Again, winds were dominantly retrograde $(\sim 50-80 \mathrm{~m} / \mathrm{s}$ at Equator $)$, but becoming prograde southward of $40^{\circ} \mathrm{S}$, with velocities of $\sim 25-50 \mathrm{~m} / \mathrm{s}$ at $75^{\circ} \mathrm{S}$. Unlike in our Nov. 2005 results, satisfactory agreement was found with GCM predictions. Simultaneous $\mathrm{mm} / \mathrm{submm}$ and $10-\mu \mathrm{m}$ observations would be desirable to understand this discrepancy.

\section{Summary}

We have obtained absolute wind and thermal profile measurements in the martian atmosphere from repeated observations of the $\mathrm{CO}(1-0)$ line with the IRAM Plateau de Bure Interferometer. Our observations have sampled several seasons (Southern summer solstice (2003), late Southern summer (2003 and 2005), late Northern summer (1999), and Northern fall equinox (2001)), as well as a variety of dust conditions (clear, regional dust storm, and global dust storm). These data are complemented by low-resolution wind measurements obtained over no less than seven oppositions from single-dish IRAM-30 m observations. Altogether, they represent a first step toward a climatology database for Mars middle atmosphere dynamics. A persistent feature is that the circulation near $50 \mathrm{~km}$ is dominated by strong retrograde winds, which typical velocities of 70-170 m/s, strongly varying seasonally, latitudinally, and longitudinally (i.e. either geographically or diurnally). Comparison of the retrieved winds and profiles with GCM predictions indicates that with the exception of clear summer solstice conditions, the models generally underpredict the general strength of the winds, do not show as much spatial structure as in the observations, and often underestimate the temperatures in the middle (20-50 km) atmosphere, particularly in the winter hemisphere. Part of the discrepancies may be, at least qualitatively, be accounted for by an improper representation of dust activity in the models. Actual temperatures warmer than expected intensify the meridional circulation and produce more intense zonal jets. Additional discrepancies are present when the amount of dust is higher than in the reference dust scenario; this is the case at $\mathrm{L}_{s} \sim 320$ in 2003 and 2005, and during the 2001 global dust storm at $\mathrm{L}_{s}=197$. Finally, the atmospheric temperature above $50 \mathrm{~km}$ is often overestimated in the GCM, a probably consequence of an inaccurate estimate of non-LTE cooling.

From the observation point of view, the ALMA array will allow observations with higher signal-to-noise, spatial and temporal resolution, providing instantaneous dynamic views of the 
martian atmosphere. This will be a starting point for data assimiliation of the CO-derived thermal structure in the Mars' middle atmosphere. A major improvement would consist to have a submm sounder on a Martian orbiter. This submm instrument would allow to determine the 3D atmospheric circulation, temperature and water cycle, day after day and perform comparative meteorology (Forget et al. 2004, Hartogh et al. 2007).

\section{Acknowledgements}

Observations were carried out with the IRAM Plateau de Bure Interferometer. IRAM is supported by INSU/CNRS (France), MPG (Germany) and IGN (Spain). This work has been supported by the French Programme National de Planétologie (PNP). 


\section{References}

Angelats i Coll, M., Forget, F., López-Valverde, M.-A., González-Galindo, F., 2005. The first Mars thermospheric general circulation model: the martian atmosphere from the ground to $240 \mathrm{~km}$. Geophys. Res. Lett., 32, doI 10.1029/2004GL021368.

Cavalié, T., Billebaud, F., Encrenaz, T., Brillet, J., Forget, F., Lellouch, E. 2008. Vertical temperature profile and mesospheric winds retrieval on Mars from CO millimeter observations. Comparison with general circulation model predictions. Astron. Astrophys., 489, 795-809

Clancy, R.T., Muhleman, D.O., Berge, G. L., 1990. Global changes in the 0-70 km thermal structure of the Mars atmosphere derived from 1975 to 1989 microwave CO spectra. J. Geophys. Res., 95, 14543-14554.

Clancy, R.T., Sandor, B.J., Wolff, M.J., Christensen, P.R., Smith, M.D., Pearl, J.C., Conrath, B.J., Wilson, R.J. (2000). An intercomparison of ground-based millimeter, MGS TES, and Viking atmospheric temperature measurements: Seasonal and interannual variability of temperatures and dust loading in the global Mars atmosphere. J. of Geophys. Res., 105, 9553-9572.

Clancy, R.T., Sandor, B.J., Moriarty-Schieven, G.H., Smith M.D., 2006. Mesospheric winds and temperatures from JCMT sub-millimeter CO lines observations during the 2003 and 2005 Mars oppositions. Paper presented at 2nd workshop on Mars Atmosphere Modelling and Observations, Granada, Spain, 27 Feb - 3 Mar. 2006. p135.

Forget, F., Hourdin, F., Fournier, R., Hourdin, C., Talagrand, O., Collins, M., Lewis, S.R., Read, P.L., Huot, J.-P., 1999. Improved general circulation models of the Martian atmosphere from the surface to above $80 \mathrm{~km}$. J. Geophys. Res., 104, 24155-24176, doi: 10.1029/1999JE001025.

Forget, F., Gonzalez-Galindo, F., Lebonnois, S., Bertaux, J.L., Montmessin, F., Quémerais, E., Reberac, A., Lopez-Valeverde, M.A., 2008. The density and temperatures of the martian upper atmosphere measured by stellar occultations with Mars Express SPICAM. J. Geophys. Res., doi:10.1029/2008JE003086, in press 
Forget, F., The Mambo Team (2004). A Submm Sounder for the Exploration of Mars. COSPAR, 35, 4103.

Gillet, V., and Lellouch, E., 1994. Does Mars' middle atmosphere really have a retrograde superrotation near Equinox. Bull. Amer. Astron. Soc., 26, 1138.

Grassi, D., Formisano, V., Forget, F.,Fiorenza, C., Ignatiev, N.I., Maturilli, A., Zasova, L.V., 2007. The martian atmosphere in the region of Hellas bassin as observed by the planetary Fourier spectrometer (PFS-MEX). Planet. Space Sci, $55,1346-1357$.

Haberle, R.M., Pollack, J.B., Barnes, J.R., Zurek, R.W., Leovy, C.B., Murphy, J.R., Lee, H., Schaeffer, J., 1993. Mars atmospheric dynamics as simulated by the NASA AMES General Circulation Model. I - The zonal-mean circulation. J. Geophys. Res., 98, 3093-3123.

Hartogh, P., Medvedev, A.S., Kuroda, T. , Saito, R., Villanueva, G, Feofilov, A.G., Kutepov, A.A. Berger, U., 2005. Description and climatology of a new general circulation model of the Martian atmosphere. J. Geophys. Res. 2005, 110, doI 10.1029/2005JEOO2498.

Hartogh, P., Medvedev, A.S., Jarchow, C. 2007. Middle atmosphere polar warmings on Mars: Simulations and study on the validation with sub-millimeter observations. Planet. Space Sci., 55, 1103-1112.

Hinson, D.P., Smith, M.D., Conrath, B.J., 2004. Comparison of atmospheric temperatures obtained through infrared sounding and radio occultation by Mars Global Surveyor. J. Geophys. Res. 109, doI 10.1029/2004JE002344.

Jegou, F. 2003. Etude de la dynamique de la moyenne atmosphère de Mars à partir de données millimétriques. Thèse Université Paris-6.

Krasnopolsky, V.A., 2003. Spectroscopic mapping of Mars CO mixing ratio: detection of north-south asymmetry. J. Geophys. Res., 108, E2, doi 10.1029/2002JE001926

Krasnopolsky, V.A., 2007. Long-term spectroscopic observations of Mars using IRTF/CSHELL: Mapping of $\mathrm{O}_{2}$ dayglow, $\mathrm{CO}$, and search for $\mathrm{CH}_{4}$. Icarus, 190, 93-102.

Lellouch, E., Rosenqvist, J., Goldstein, J.J., Bougher, S.W., Paubert, G., 1991a. 
First absolute wind measurements in the middle atmosphere of Mars. Astrophys. J., 383, 401-406.

Lellouch, E., Paubert, G., Encrenaz, T., 1991b. Mapping of CO millimeter-wave lines in Mars' atmosphere. The spatial variability of carbon monoxide on Mars. Planet. Space Sci. 39, 219-224.

Lewis, S.R., Collins, M., Read, P. L., Forget, F., Hourdin, F., Fournier, R., Hourdin, C., Talagrand, O., Huot, J.-P, 1999. A climate database for Mars. J. Geophys. Res., 104, 24177-241794, doi: 10.1029/1999JE001024.

Lillis, R.J., Bougher, S.W., Mitchell, D.L., Brain, D.A., Lin, R.P., Acua, M.H., 2008 Continuous monitoring of nightside upper thermospheric mass densities in the martian southern hemisphere over 4 martian years using electron reflectometry. Icarus, 194, 562-574.

Moreno, R., Marten, A., Biraud, Y., Bézard, B; Lellouch, E., Paubert, G., Wild, W., 2001. Jovian stratospheic temperature during the two months following the impacts of comet Shoemaker-Levy 9. Planet. Space Sci., 49, 473-486.

Muhleman, D.O., and Berge, G.L., 1991. Observations of Mars, Uranus, Neptune, Io, Europa, Ganymede and Callisto at a wavelength of $2.66 \mathrm{~mm}$. Icarus, 92, 263-272.

Rudy, D.J, Muhleman, D.O., Berge, G.L., Jakosky, B.M., Christensen, P.R., 1987. Mars: VLA observations of the northern hemisphere and the north polar region at wavelengths of 2 and $6 \mathrm{~cm}$. Icarus, 71, 159-177.

Smith, M.D., 2002. The annual cycle of water on Mars as observed by the Thermal Emission Spectrometer. J. Geophys. Res., 107, doi:10.1029/2001JE001522.

Smith, M.D., 2004. Interannual variability in TES atmospheric observations of Mars during 1999-2003. Icarus 167, 148-165.

Smith, M.D., 2006. TES atmospheric temperature, aerosol optical depth, and water vapor observations 1999-2004. Paper presented at 2nd workshop on Mars Atmosphere Modelling and Observations, Granada, Spain, 27 Feb - 3 Mar. 2006. p211

Sonnabend, G., Wirtz, D., Vetterle, V, and Schieder, R., 2005. High-resolution observations of Martian non-thermal $\mathrm{CO}_{2}$ emission near $10 \mu \mathrm{m}$ with a new tuneable 
heterodyne receiver. Astron. Astrophys. 435, 1181-1184.

Sonnabend, G., Sornig, M., Klötz, P.J., Schieder, R.T., and Fast, K.E., 2006. High spatial resolution mapping of Mars mesospheric zonal winds by infrared heterodyne spectroscopy of $\mathrm{CO}_{2}$. Geophys. Res. Lett., 33, L18201., doi: 10.1029/2006GL026900.

Strausberg, M.J., Wang, H., Richardson, M.I., Ewald, S.P., Toigo, A.D., 2005. Observations of the initiation and evolution of the 2001 Mars global dust storm. J. Geophys. Res. 110, E02006., doi:10.1029/2004JE002361.

Théodore, B., Lellouch, E., Chassefiere, E., Hauchecorne, A., 1993. Solstitial temperature inversions in the Martian middle atmosphere. Observational clues and 2-D modeling, Icarus, 105, 512-528.

Wang, H., Zurek, R.W., Richardson, M.I., 2005. Relationship between frontal dust storms and transient eddy activity in the northern hemisphere of Mars as observed by Mars Global Surveyor. J. Geophys. Res. 110, E07005., doi:10.1029/2005JE002423.

Wang, H., 2007. Dust storms originating in the northern hemisphere during the third mapping year of Mars Global Surveyor. Icarus, 189, 325-343.

Wilson, R.J., Lewis, S.R., Montabone, L., and Smith, M.D., 2008. Influence of water ice clouds on Martian tropical atmospheric temperatures, Geophys. Res. Lett., 35, L07202. doi:10.1029/2007GL032405 


\section{Figure captions}

Fig. 1 Example of continuum visibilities measured with the PdBI on December 16, 2003 at 112.271 GHz. This frequency corresponds to the LSB frequency for the CO(1-0) tuning. Several Mars continuum models, with three different values of the surface roughness, $\left(0^{\circ}, 15^{\circ}\right.$ and $30^{\circ}$ ) are compared with the raw visibilities. The inset is a zoom on the near-zero amplitudes, particularly sensitive to the surface roughness. The best estimate of the surface roughness is $15^{\circ}$.

Fig. 2 Example of spectra observed in Sept. 2003 at eastern and western equatorial limb positions, after removal of a polynomial baseline over $\pm[1-10 \mathrm{~km} / \mathrm{s}]$. The solid and dotted vertical bars show the respective line core positions. Different Doppler shifts on the two sides are clearly visible. Note that the Doppler shift due to the planet rotation $( \pm 240 \mathrm{~m} / \mathrm{s}$ at equator, unconvolved) has not been removed here.

Fig. 3 Wind Weighting function (WWF) in the $\mathrm{CO}(1-0)$ line at limb (solid line) and nadir (dashed line). The WWF is defined as the frequency-averaged contribution function, weighted by the local slope of the spectrum over the fitting interval $( \pm 1 \mathrm{~km} / \mathrm{s})$.

Fig. 4 Sept. 2003 PdBI Observed Doppler winds from CO(1-0) line (6). The Mars' rotation velocity has been corrected. The North pole has been rotated to top.

Fig. 5 Sept. 2003 PdBI modelled wind. Expected Doppler winds, based on GCM predictions

Fig. 6 Sept. 2003 PdBI Map of CO line core. Black lines: observations. Red lines: Line profile expected from GCM predictions. Green lines: Fit of observations with retrieved thermal profiles.

Fig. 7 Comparison of beam-convolved retrieved (green line) and GCM (red lines) thermal profile (red lines), for the Sept. 2003 period. 
Fig. 8 May 1999 PdBI observations. Top left. Observed Doppler winds from CO(1-0) line, corrected for Mars' rotation. The North pole has been rotated to top. Top right. Expected Doppler winds, based on GCM predictions. Bottom left. Map of CO line core. Black lines: observations. Red lines: Line profile expected from GCM predictions. Green lines: Fit of observations with retrieved thermal profiles. Bottom right. Comparison of beam-convolved retrieved (green line) and GCM (red lines) thermal profile (red lines).

Fig. 9 Expected projected Doppler wind for the geometry of the May 1999 observation according to GCM predictions - in the absence of beam convolution.

Fig. 10 Same as Fig. 8 for Dec. 2003 PdBI observations. Note that the color scales are different in the top two figures.

Fig. 11 Same as Fig. 8 for Nov. 2005 PdBI observations. Note that the color scales are different in the top two figures.

Fig. 12 Same as Fig. 8 for July 2001 PdBI observations. Note that the color scales are different in the top two figures.

Fig. 13 Doppler wind measurements (corrected for rotation, positive for recession) based on $\mathrm{CO}(2-1)$ observations with the IRAM-30m telescope. One- $\sigma$ error bars are indicated in parentheses. The green circle shows the 10.4" telescope beam. In addition to the 6 epochs shown here, wind measurements obtained in 1988 are shown in Lellouch et al. (1991a), and observations obtained in 2001 and 2005 are presented in Cavalié et al. (2008).

Fig. 14 Deprojected Doppler wind velocities (positive values indicates retrograde flows) at Equator, mid-Northern $(\sim 20-50 \mathrm{~N})$ and mid-Southern $(\sim 20-50 \mathrm{~S})$ latitudes as a function of $\mathrm{L}_{s}$, based on the IRAM 30-m and IRAM PdBI dataset (see Table 3). Red points refer to the morning limb (sky east, martian west); blue crosses refer to the evening limb (sky west, martian west). The expected zonal wind (at $\mathrm{z}=50 \mathrm{~km}$ ) from the GCM, is also indicated (solid lines, 
same color as data). 
Table 1: Observation parameters at PdBI

\begin{tabular}{|c|c|c|c|c|c|c|c|}
\hline Date & $\mathrm{L}_{s}$ & $<\mathrm{UT}>$ & Diameter & \multicolumn{2}{|c|}{ Beam } & $<\mathrm{H}_{2} \mathrm{O}>$ pwv & $<$ Atm. Phase $>$ \\
\hline & & & & & $\mathrm{PA}^{a}$ & & \\
& & & $\left({ }^{\prime \prime}\right)$ & $\left({ }^{\prime \prime}\right)$ & $\left({ }^{\circ}\right)$ & $(\mathrm{mm})$ & $\left({ }^{\circ}\right)$ \\
\hline $15 / 05 / 99$ & 139 & $23 \mathrm{~h} 00$ & 15.64 & - & - & 3 & 30 \\
$24 / 05 / 99$ & 143 & $21 \mathrm{~h} 00$ & 14.94 & - & - & 4.5 & 25 \\
$25 / 05 / 99$ & 143 & $21 \mathrm{~h} 45$ & 14.85 & - & - & 3.5 & 34 \\
$04 / 06 / 99$ & 149 & $21 \mathrm{~h} 00$ & 13.89 & - & - & 4 & 45 \\
\hline $23 / 05 / 99$ & 143 & $21 \mathrm{~h} 00$ & $15.000^{b}$ & $5.26^{*} 2.81$ & -13 & - & - \\
$17 / 07 / 01$ & 196 & $21 \mathrm{~h} 10$ & 18.870 & $9.41^{*} 4.81$ & 154 & 7 & 34 \\
$17 / 09 / 03$ & 262 & $22 \mathrm{~h} 40$ & 23.134 & $8.30^{*} 5.79$ & 14 & 4 & 15 \\
$16 / 12 / 03$ & 317 & $18 \mathrm{~h} 50$ & 9.572 & $3.85^{*} 3.41$ & 56 & 2.5 & 11 \\
$11 / 11 / 05$ & 322 & $23 \mathrm{~h} 00$ & 19.561 & $6.07^{*} 5.72$ & 51 & 4.5 & 22 \\
\hline
\end{tabular}

${ }^{a}$ PA denotes the position angle of the elliptical synthezised beam of the interferometer.

${ }^{b}$ Since in 1999 four observations periods were recorded, the uv-table were merged after re-scaling to an average angular diameter of 15.0 arcsec.

Table 2: Continuum roughness measurements

\begin{tabular}{|c|c|}
\hline Date & $\begin{array}{c}\text { Surface roughness } \\
\left({ }^{\circ}\right)\end{array}$ \\
\hline $24 / 05 / 99$ & $15-30$ \\
$17 / 09 / 03$ & $0-30$ \\
$16 / 12 / 03$ & $0-15$ \\
$11 / 11 / 05$ & $15-30$ \\
\hline
\end{tabular}


Table 3: Overview of Doppler wind measurements from IRAM 30-m and PdBI

\begin{tabular}{|c|c|c|c|c|c|c|c|c|c|c|c|c|}
\hline \multirow[t]{3}{*}{ Date } & \multirow[t]{3}{*}{$\mathrm{L}_{s}$} & \multirow[t]{3}{*}{$\mathrm{LT}^{1}$} & \multicolumn{8}{|c|}{ Deprojected zonal wind ${ }^{2}(\mathrm{~m} / \mathrm{s})$} & \multirow[t]{3}{*}{$\mathrm{V}_{e q}^{3}$} & \multirow[t]{3}{*}{$\tau_{\text {dust }}^{5}$} \\
\hline & & & \multicolumn{2}{|c|}{ Equator } & \multicolumn{3}{|c|}{ Mid-Northern lat. } & \multicolumn{3}{|c|}{ Mid Southern lat. } & & \\
\hline & & & East $^{4}$ & West $^{4}$ & East & West & Lat & East & West & Lat & & \\
\hline $23 / 09 / 88^{a}$ & 279 & $12 \mathrm{~h} 15$ & $127(39)$ & $87(40)$ & $0(43)$ & $136(40)$ & $25 \mathrm{~N}$ & $100(62)$ & $180(52)$ & $35 \mathrm{~S}$ & $110(55)$ & - \\
\hline $20 / 12 / 90^{b}$ & 352 & $10 \mathrm{~h} 55$ & $79(33)$ & $105(24)$ & & & & & & & $115(50)$ & - \\
\hline $31 / 12 / 92^{b}$ & 19 & $12 \mathrm{~h} 30$ & $98(08)$ & $87(08)$ & $120(13)$ & $69(10)$ & $40 \mathrm{~N}$ & $121(11)$ & $95(11)$ & $35 \mathrm{~S}$ & $110(15)$ & - \\
\hline $07 / 04 / 97^{c}$ & 101 & $10 \mathrm{~h} 45$ & $214(25)$ & $112(20)$ & & & & & & & $170(70)$ & - \\
\hline $27 / 04 / 99^{c}$ & 130 & $11 \mathrm{~h} 50$ & $85(16)$ & $135(10)$ & $58(23)$ & $75(25)$ & $40 \mathrm{~N}$ & $187(34)$ & $65(19)$ & $40 \mathrm{~S}$ & $130(30)$ & 0.1 \\
\hline $23 / 05 / 99$ & 143 & $10 \mathrm{~h} 30$ & $41(21)$ & $105(13)$ & $41(21)$ & $128(17)$ & $27 \mathrm{~N}$ & $131(31)$ & $137(17)$ & $37 \mathrm{~S}$ & & 0.1 \\
\hline $15 / 06 / 01^{d}$ & 180 & $12 \mathrm{~h} 00$ & $56(05)$ & $82(03)$ & $41(06)$ & $12(07)$ & $48 \mathrm{~N}$ & $53(07)$ & $44(07)$ & $50 \mathrm{~S}$ & $65(30)$ & 0.2 \\
\hline $17 / 07 / 01$ & 196 & 10h30 & $0(17)$ & $47(17)$ & $-109(22)$ & $-202(27)$ & $60 \mathrm{~N}$ & $160(88)$ & $-46(46)$ & $60 \mathrm{~S}$ & & 4.0 \\
\hline $15 / 08 / 03$ & 241 & $12 \mathrm{~h} 55$ & $113(09)$ & $69(08)$ & $-9(32)$ & $-49(32)$ & $40 \mathrm{~N}$ & $-32(37)$ & $46(28)$ & $35 \mathrm{~S}$ & $80(25)$ & 0.3 \\
\hline & & & & & & & & $78(62)$ & $-127(62)$ & $60 \mathrm{~S}$ & & \\
\hline 01/09/03 & 252 & $12 \mathrm{~h} 10$ & $110(06)$ & $124(08)$ & $127(10)$ & $104(09)$ & $30 \mathrm{~N}$ & $102(06)$ & 181(10) & $30 \mathrm{~S}$ & $130(20)$ & 0.3 \\
\hline & & & & & & & & $69(08)$ & $177(10)$ & $60 \mathrm{~S}$ & & \\
\hline $17 / 09 / 03$ & 262 & $10 \mathrm{~h} 50$ & $105(11)$ & $80(22)$ & $-167(96)$ & $-125(55)$ & $52 \mathrm{~N}$ & $110(12)$ & $-178(31)$ & $70 \mathrm{~S}$ & & 0.3 \\
\hline $16 / 12 / 03$ & 317 & $9 \mathrm{~h} 00$ & $16(36)$ & $248(33)$ & - & $81(31)$ & $35 \mathrm{~N}$ & $166(44)$ & $193(28)$ & $47 \mathrm{~S}$ & & 0.5 \\
\hline $31 / 10 / 05^{d}$ & 316 & $12 \mathrm{~h} 25$ & $83(04)$ & $151(07)$ & $51(04)$ & $195(06)$ & $40 \mathrm{~N}$ & $94(06)$ & $124(07)$ & $45 \mathrm{~S}$ & $105(35)$ & 0.3 \\
\hline $11 / 11 / 05$ & 322 & $11 \mathrm{~h} 45$ & $272(27)$ & $154(37)$ & $-202(52)$ & $-46(30)$ & $40 \mathrm{~N}$ & $68(23)$ & $59(31)$ & $60 \mathrm{~S}$ & & 0.2 \\
\hline
\end{tabular}

${ }^{1}$ Sub-earth point local time

${ }^{2}$ Deprojected zonal wind from beam position (see text). A positive (resp. negative) value indicates a locally retrograde (resp. prograde) flow.

${ }^{3}$ Best fit equatorial velocity (positive for retrograde), assuming solid-body rotation.

4 Astronomical convention. "East limb" corresponds to martian western (morning) limb.

${ }^{5}$ Averaged dayside TES and THEMIS derived dust opacity at $1075 \mathrm{~cm}^{-1}$ from Smith et al. 2004 and Lillis et al. 2008. References: ${ }^{a}$ Lellouch et al. 1991a, ${ }^{b}$ Gillet and Lellouch 1994, ${ }^{c}$ Jegou (2003), ${ }^{d}$ Cavalié et al., 2008. 


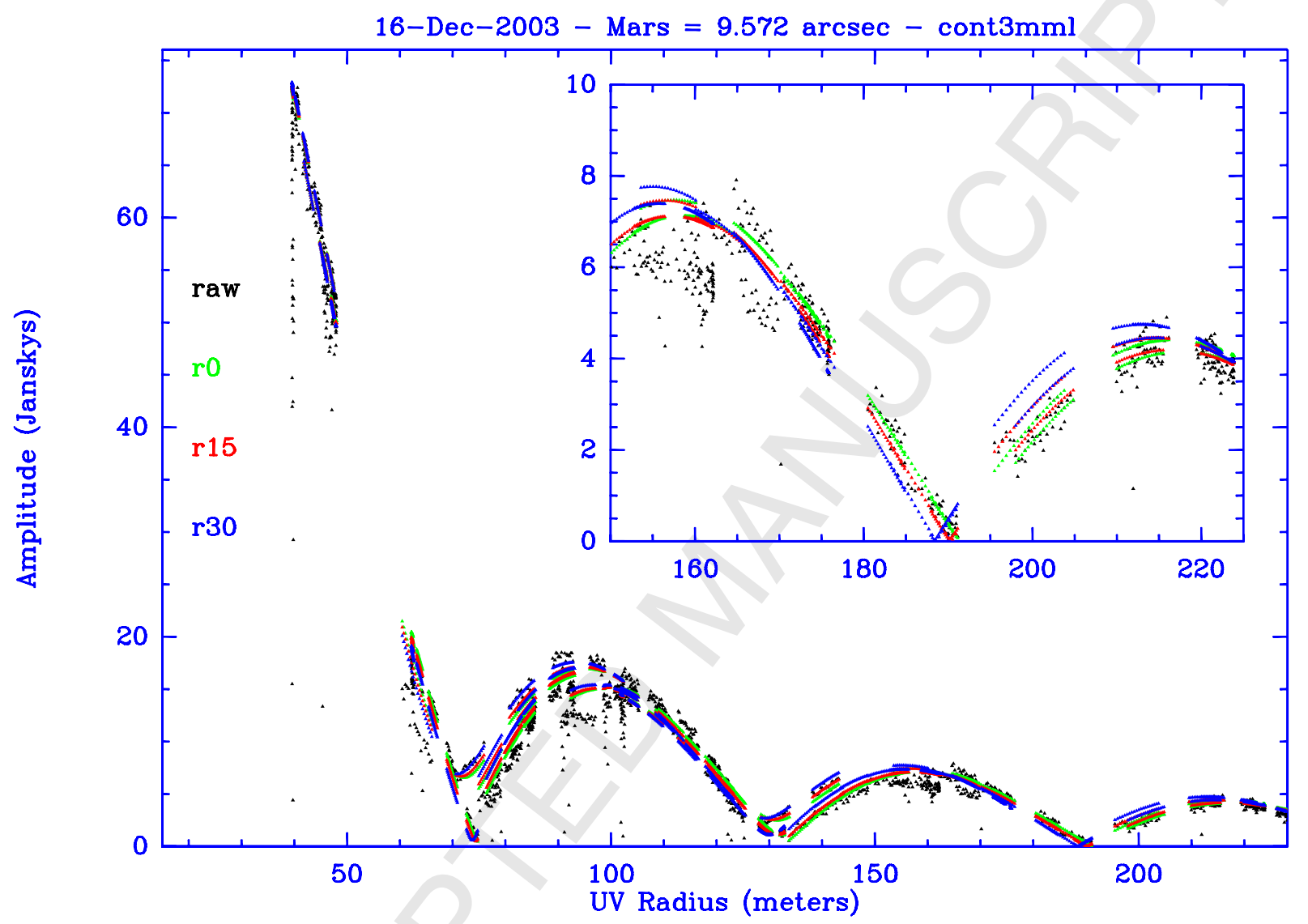

Figure 1: 


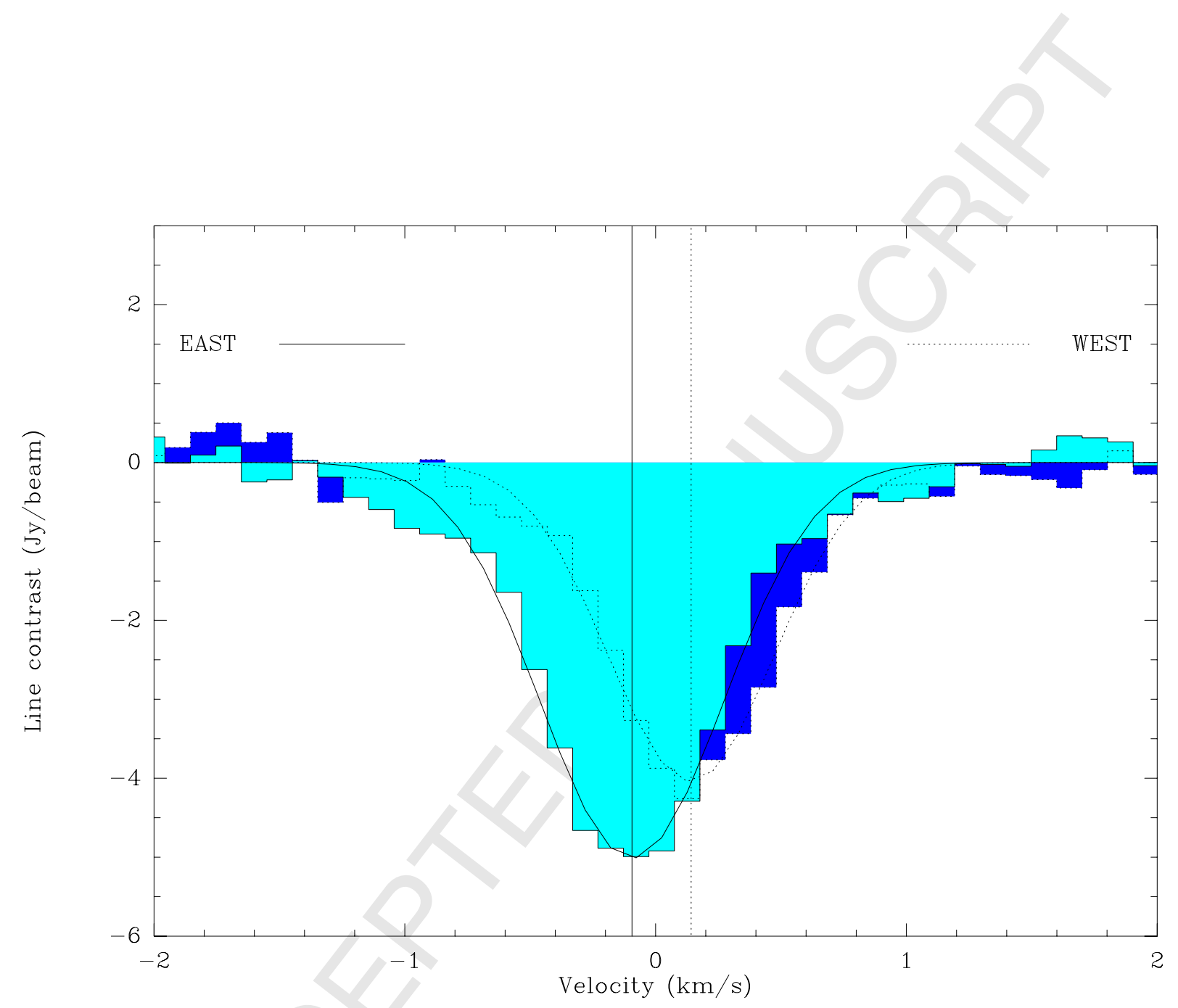

Figure 2: 


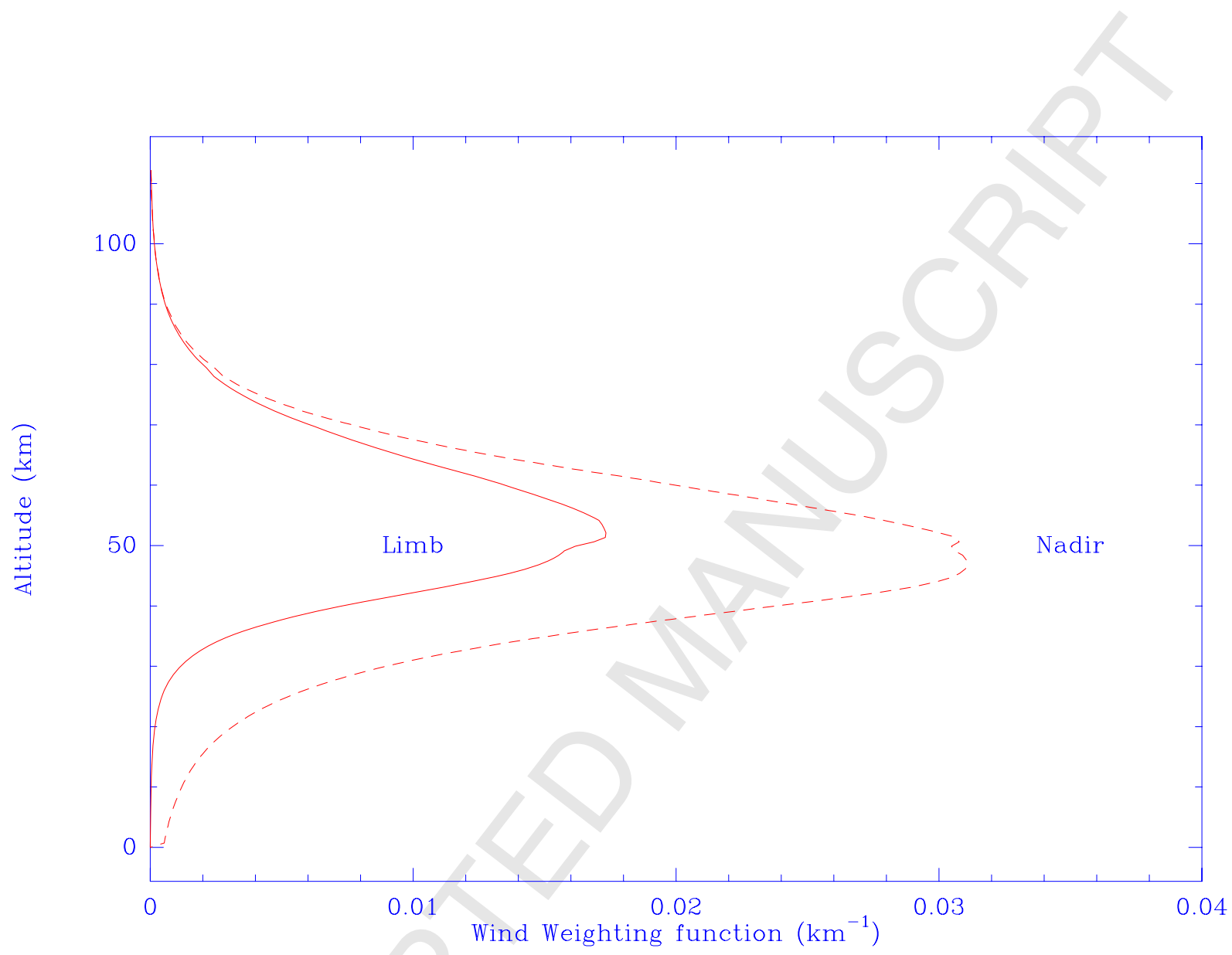

Figure 3: 


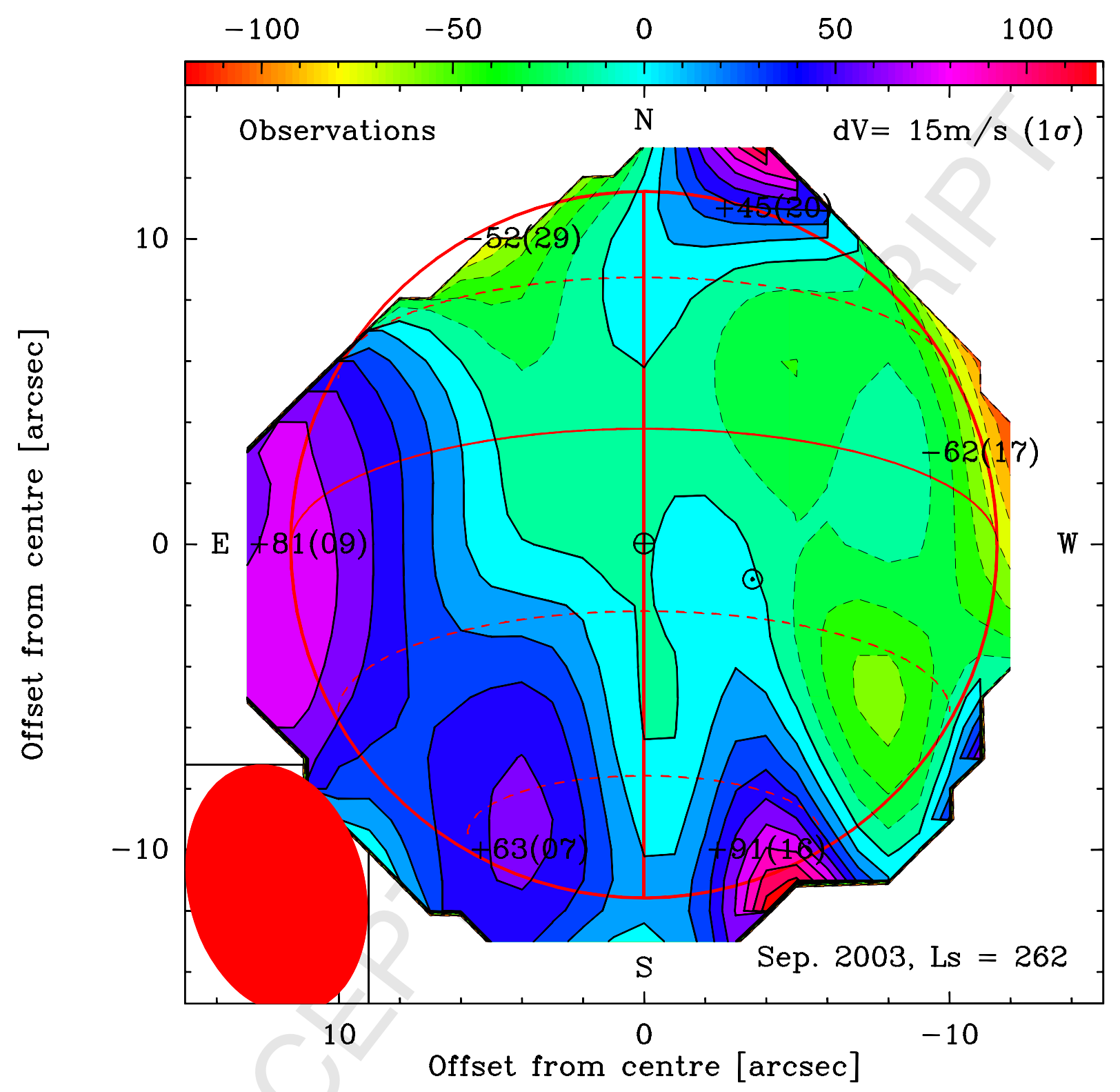

Figure 4: 


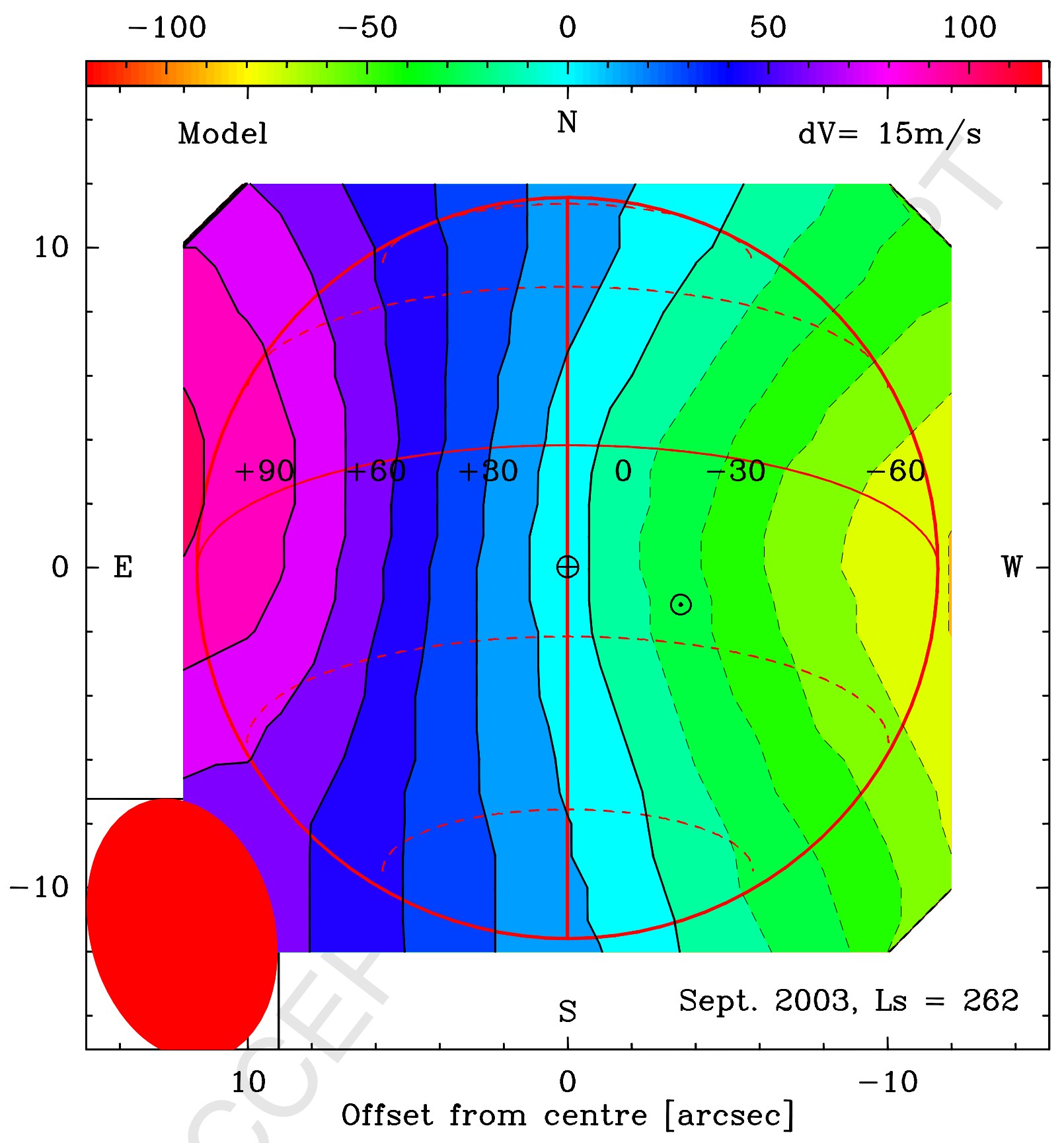

Figure 5: 


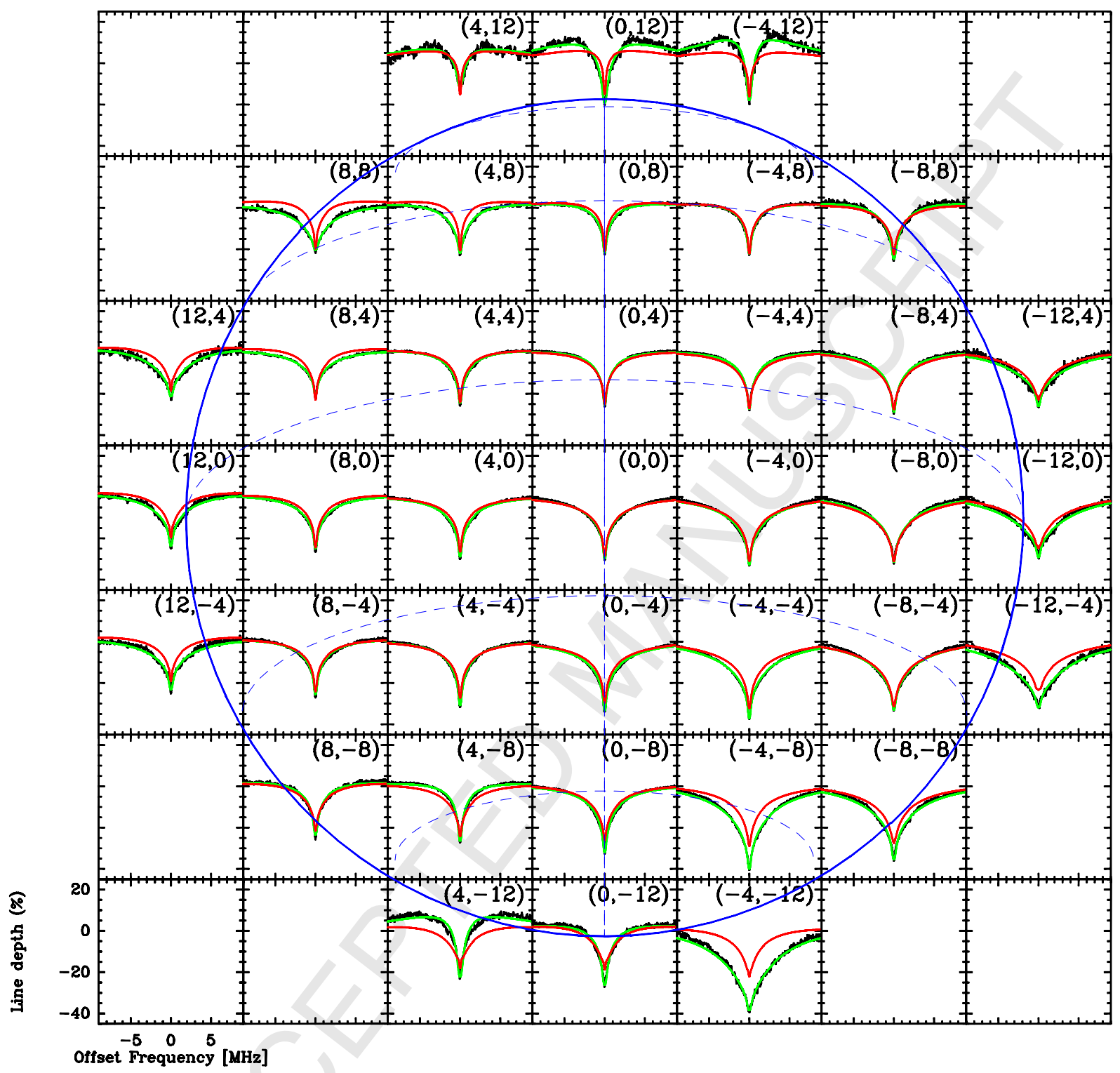

Figure 6: 


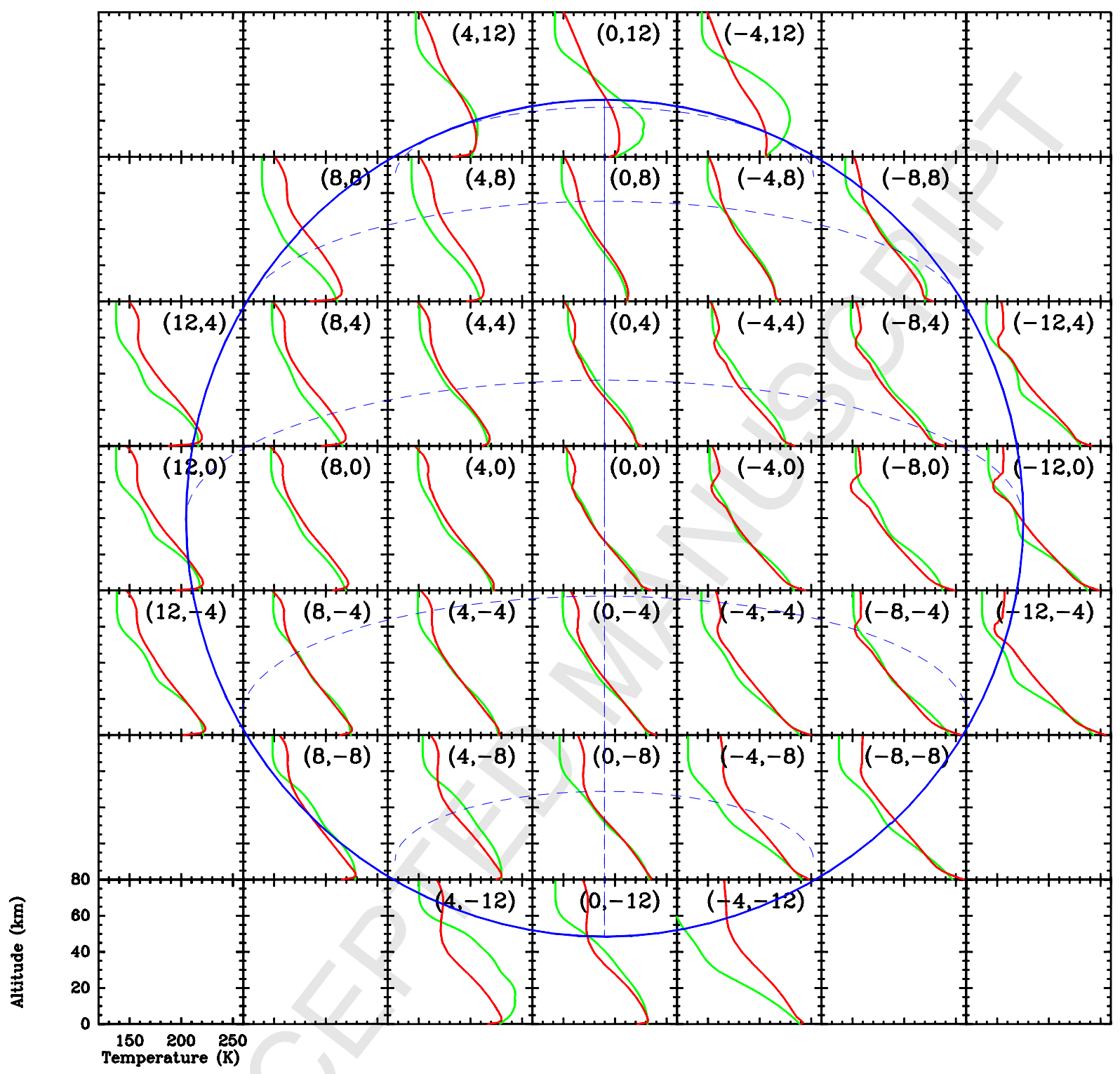

Figure 7: 

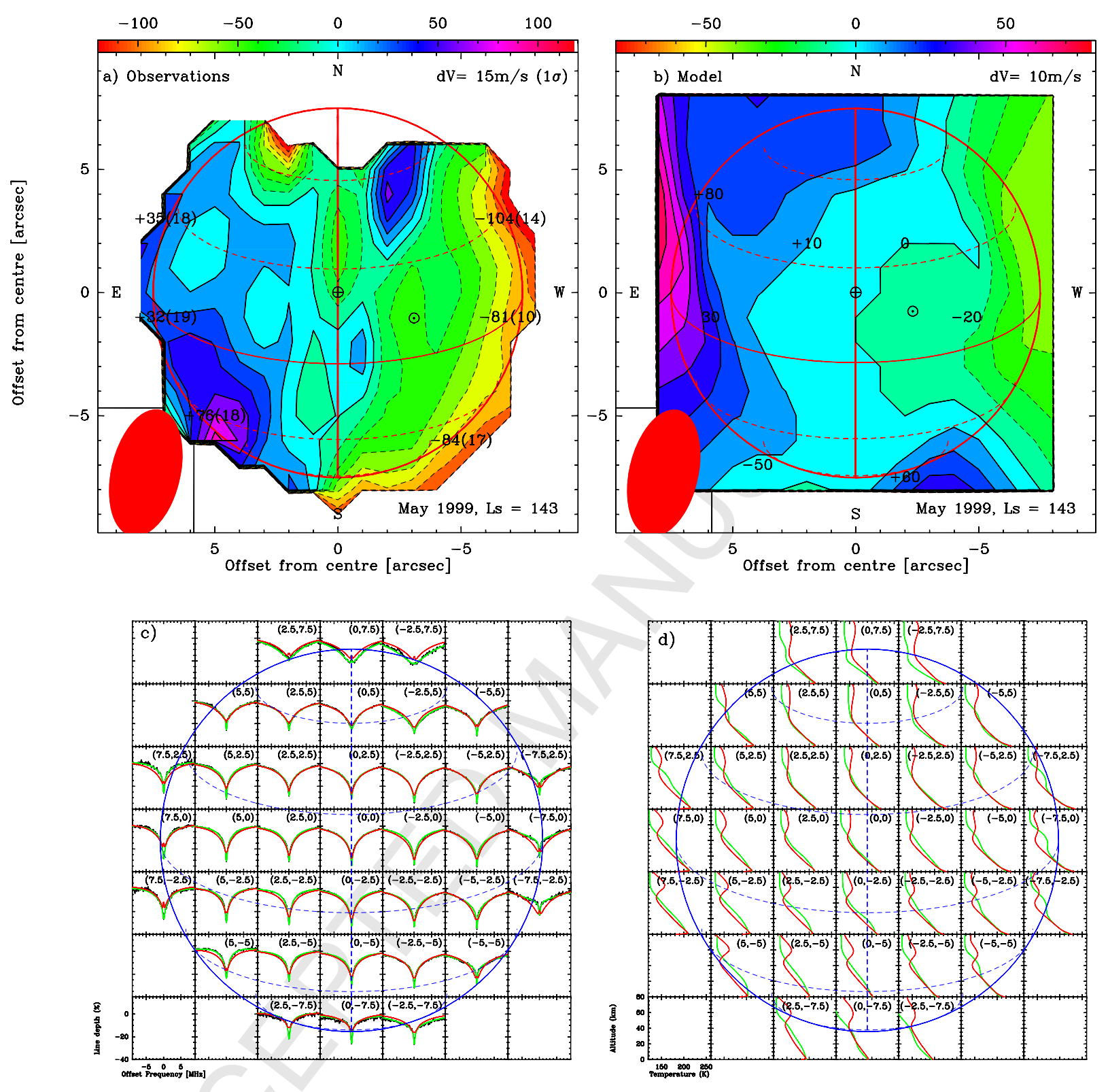

Figure 8: 


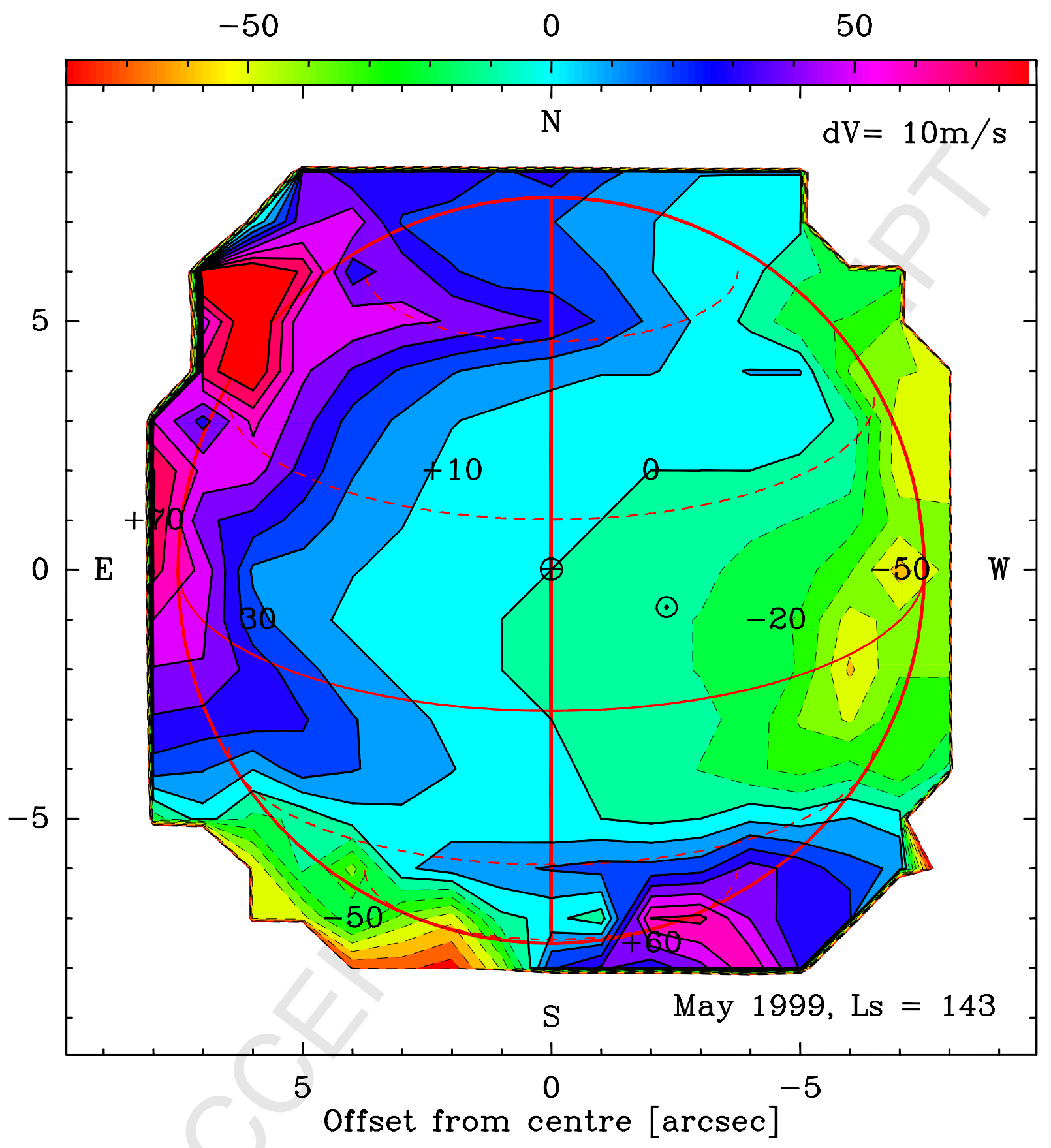

Figure 9: 

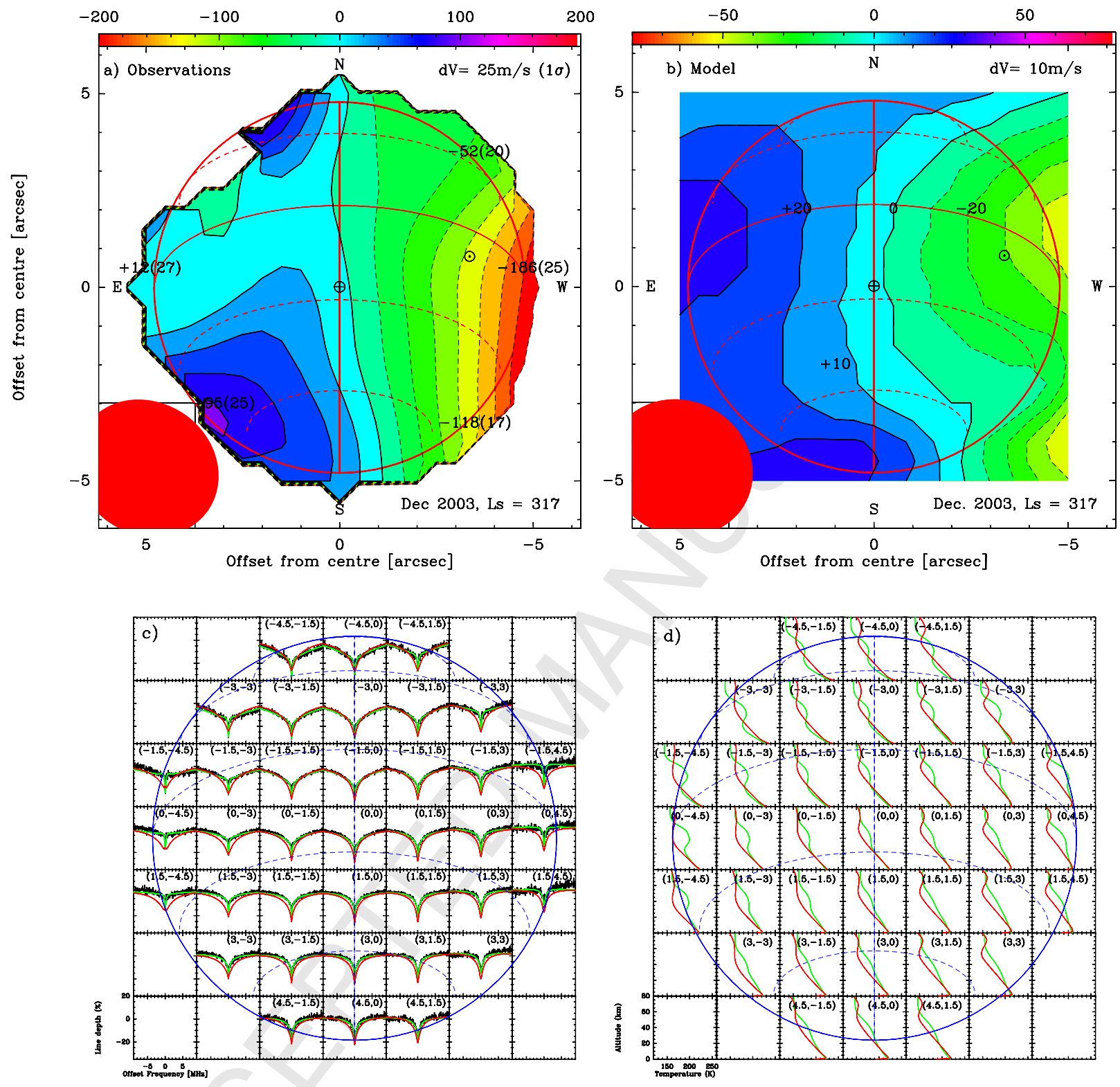

Figure 10: 

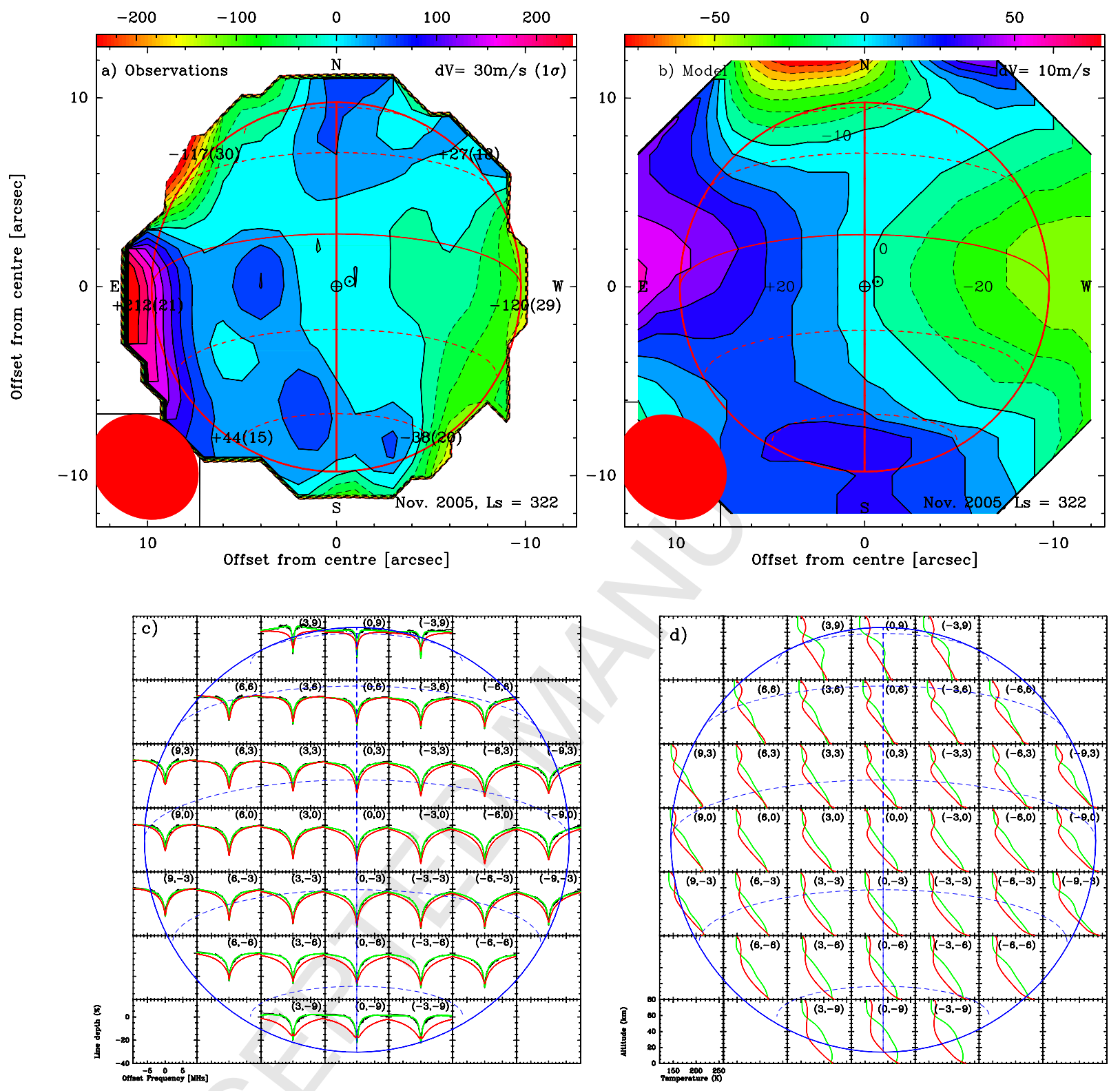

Figure 11: 

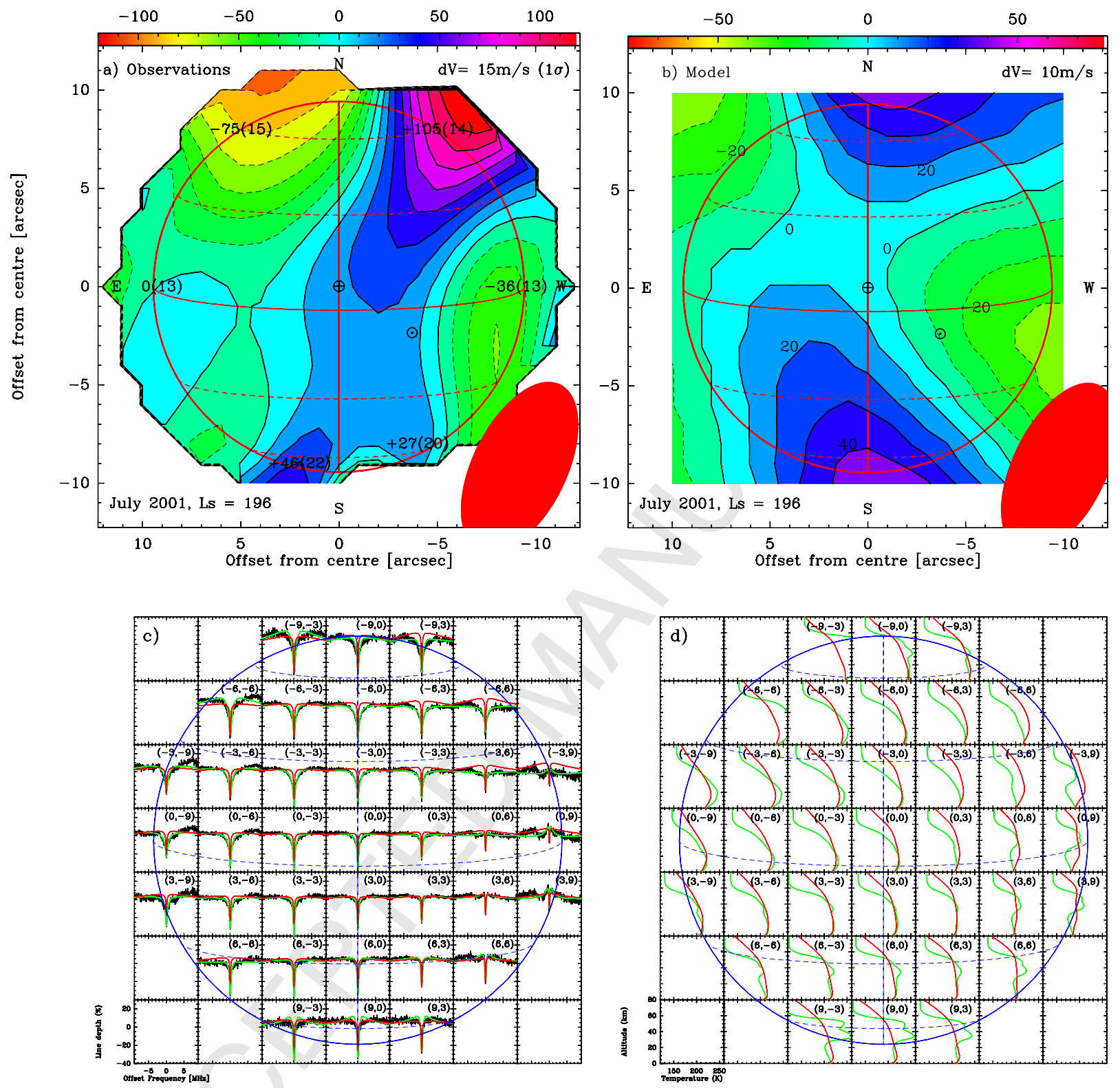

Figure 12: 


\section{ACCEPTED MANUSCRIPT}
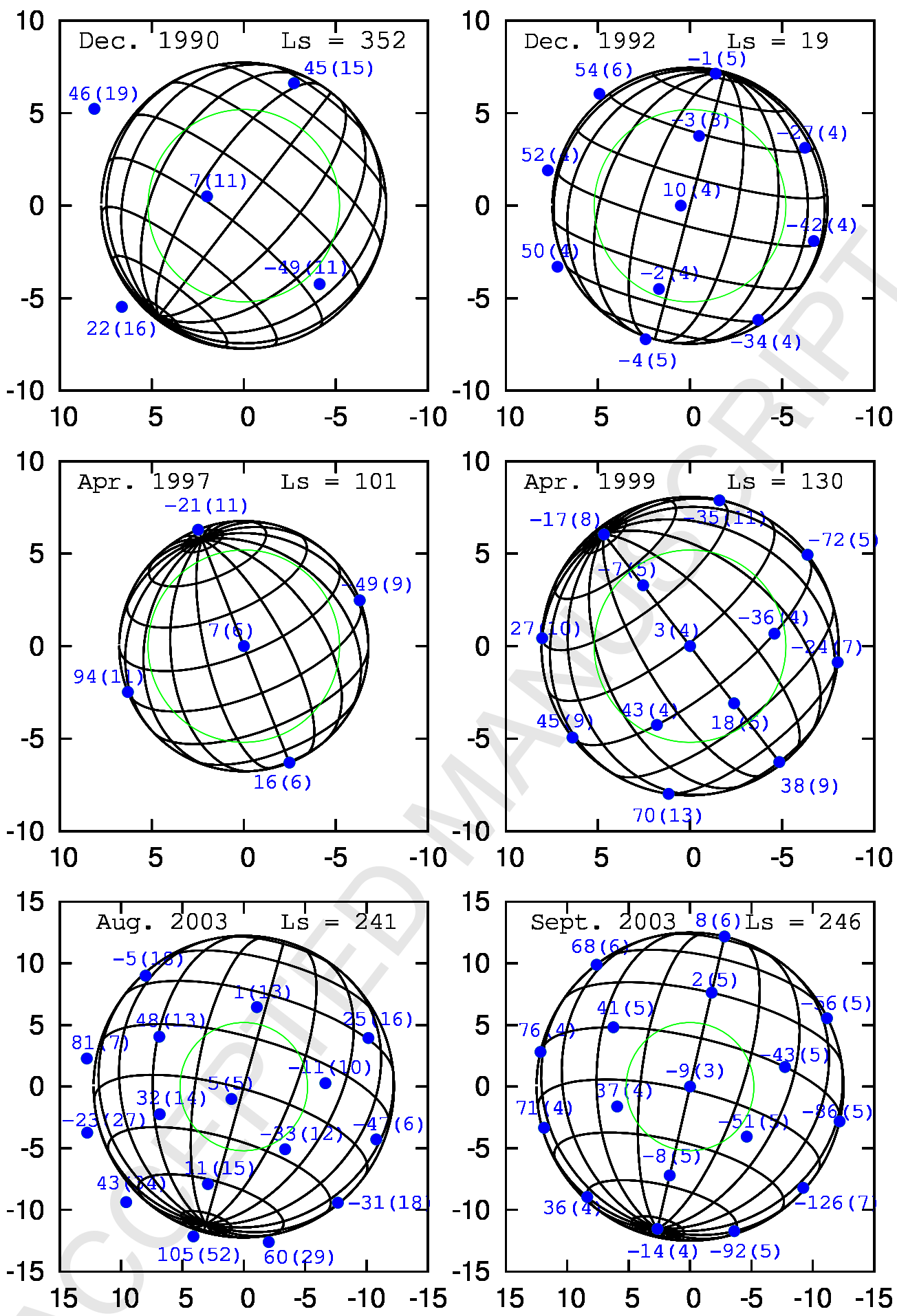

Figure 13: 


\section{ACCEPTED MANUSCRIPT}
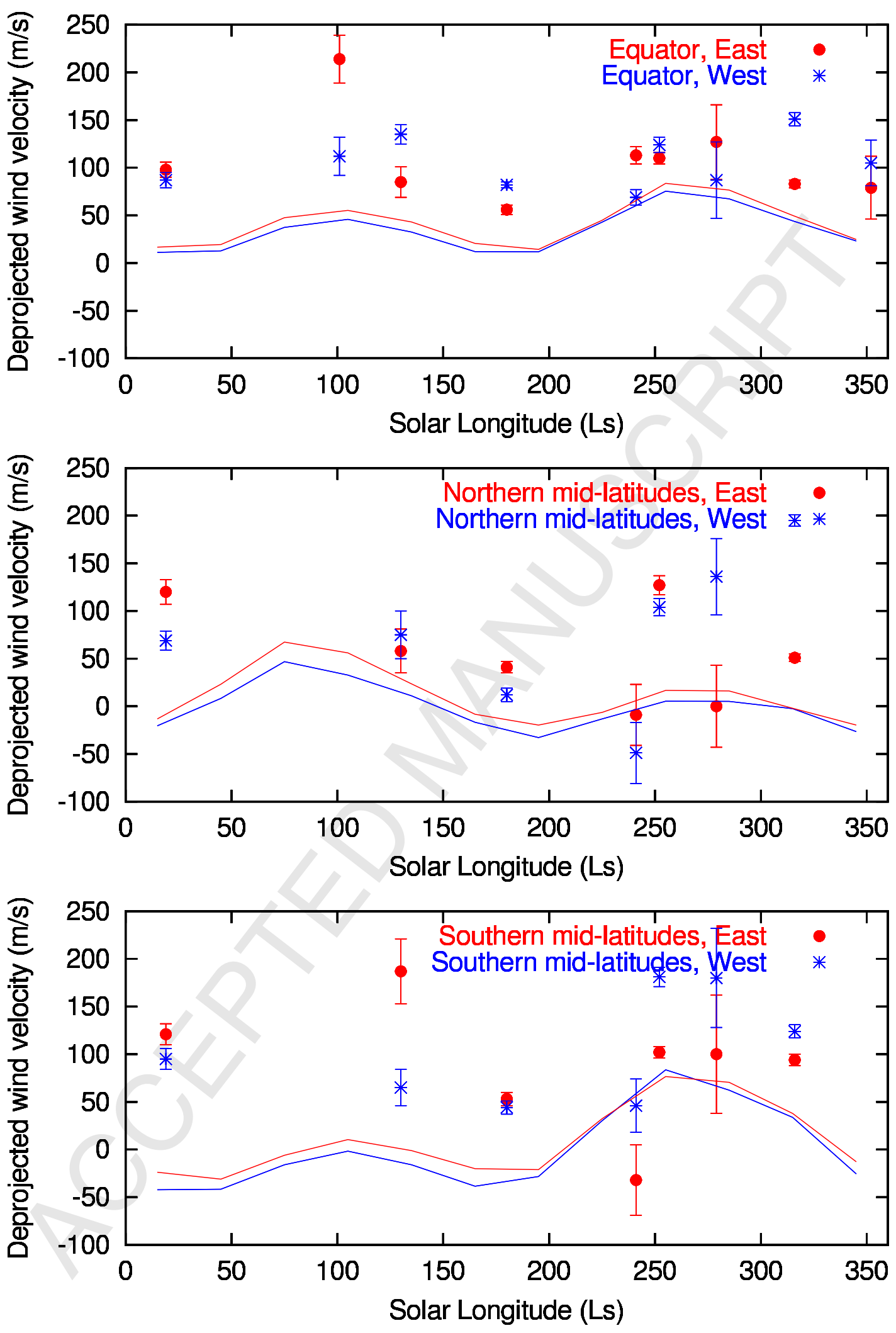

Figure 14: 